\title{
Hydrodynamic drag reduction of shear-thinning liquids in superhydrophobic textured microchannels
}

\author{
Anvesh Gaddam $^{1}$ (i) $\cdot$ Himani Sharma ${ }^{2} \cdot$ Ratan Ahuja $^{3} \cdot$ Stefan Dimov ${ }^{1} \cdot$ Suhas Joshi ${ }^{3} \cdot$ Amit Agrawal $^{3}$
}

Received: 23 April 2021 / Accepted: 19 July 2021 / Published online: 10 August 2021

(c) The Author(s) 2021

\begin{abstract}
Super-hydrophobic textured surfaces reduce hydrodynamic drag in pressure-driven laminar flows in micro-channels. However, despite the wide usage of non-Newtonian liquids in microfluidic devices, the flow behaviour of such liquids was rarely examined so far in the context of friction reduction in textured super-hydrophobic micro-channels. Thus, we have investigated the influence of topologically different rough surfaces on friction reduction of shear-thinning liquids in micro-channels. First, the friction factor ratio (a ratio of friction factor on a textured surface to a plain surface) on generic surface textures, such as posts, holes, longitudinal and transverse ribs, was estimated numerically over a range of Carreau number as a function of microchannel constriction ratio, gas fraction and power-law exponent. Resembling the flow behaviour of Newtonian liquids, the longitudinal ribs and posts have exhibited significantly less flow friction than the transverse ribs and holes while the friction factor ratios of all textures has exhibited non-monotonic variation with the Carreau number. While the minima of the friction factor ratio were noticed at a constant Carreau number irrespective of the microchannel constriction ratio, the minima have shifted to a higher Carreau number with an increase in the power-law index and gas fraction. Experiments were also conducted with aqueous Xanthan Gum liquids in micro-channels. The flow enhancement (the flow rate with superhydrophobic textures with respect to a smooth surface) exhibited a non-monotonic behaviour and attenuated with an increase in power-law index tantamount to simulations. The results will serve as a guide to design frictionless micro-channels when employing non-Newtonian liquids.
\end{abstract}

Keywords Carreau $\cdot$ Friction factor $\cdot$ Femtosecond laser $\cdot$ LIPSS $\cdot$ Shear-thinning $\cdot$ Super-hydrophobic $\cdot$ Xanthan Gum

$\begin{array}{ll}\text { Abbreviations } \\ \text { CA } & \text { Contact angle } \\ \mathrm{Ca} & \text { Capillary number } \\ \mathrm{Cu} & \text { Carreau number } \\ \text { FFR } & \text { Friction factor ratio } \\ \text { GF } & \text { Gas fraction } \\ \text { LGI } & \text { Liquid-gas interface } \\ \text { LR } & \text { Longitudinal ribs } \\ \text { Re } & \text { Reynolds number } \\ \text { SH } & \text { Super-hydrophobic } \\ \text { SLI } & \text { Solid-liquid interface }\end{array}$

Anvesh Gaddam

anvesh.gaddam@gmail.com

1 Department of Mechanical Engineering, University of Birmingham, Edgbaston, Birmingham B15 2TT, UK

2 Department of Chemical and Biomolecular Engineering, University of Notre Dame, Notre Dame, IN 46556, USA

3 Department of Mechanical Engineering, Indian Institute of Technology Bombay, Mumbai 400076, India
STL Shear-thinning liquid

TR Transverse ribs

XG Xanthan Gum

\section{Introduction}

Super-hydrophobic (SH) micro- and nano-textured surfaces have been a subject of significant interest in a plethora of applications (Geyer et al. 2020; Sharma et al. 2018; Hwang et al. 2018; Jiang et al. 2020; Gaddam et al. 2021; Chen et al. 2019), where the existence of a heterogeneous wetting state namely the Cassie-Baxter state helps in achieving the desired functionality. Similarly, in flow settings where SH-textured surfaces are involved, particularly in microscale laminar flows, the Cassie-Baxter state helps in hydrodynamic drag reduction ( $\mathrm{Li}$ et al. 2017; Ko et al. 2020) and enhanced thermo-hydraulic performance (Dilip et al. 2018; Sharma et al. 2020). Since the velocity slip induced by the entrapped gas pockets on an SH-textured surface reduces the 
pressure drop, such surfaces alleviate the pumping power requirement in microfluidic devices (Davis and Lauga 2009). However, most of the microfluidic devices operate with non-Newtonian liquids, such as blood (Laxmi et al. 2020), mucus (Elberskirch et al. 2019), polymeric liquids (Raoufi et al. 2019) and even colloidal suspensions (Liu et al. 2020). Despite the importance of such non-Newtonian liquids in microfluidics, the hydrodynamic drag reduction of these liquids is rarely investigated in the context of pressure-driven flow through SH-textured micro-channels. Therefore, it is essential to understand the flow behaviour and concomitant influence of geometrically different $\mathrm{SH}$ surface textures on flow friction in micro-channels.

The slippage properties of an $\mathrm{SH}$ surface are characterised by apparent slip length. This is defined as the distance where the no-slip boundary condition is satisfied when the tangent of the linear velocity profile is extrapolated near the wall. However, since it is difficult to measure the apparent slip length experimentally, the effective slip length is defined to characterise slippage properties of geometrically different $\mathrm{SH}$ surface textures (Lauga and Stone 2003). Ever since the pioneering work by Philip et al. (1972) to characterise slippage properties on SH surfaces between two parallel plates, most of the research efforts have focused on finding analytical expressions for the effective slip length of Newtonian liquids on generic SH surface textures, such as longitudinal ribs, transverse ribs, posts, and holes (Lauga and Stone 2003; Ybert et al. 2007; Teo and Khoo 2009; Chen et al. 2020). Since the viscosity difference between water and air is about two orders in magnitude, most of these investigations assumed that the liquid-gas interface as a shear-free boundary. Several numerical studies were also undertaken to characterise the effective slip length involving inertial and microchannel constriction effects (Sharma et al. 2020, 2019; Cheng et al. 2009; Game et al. 2017). The effective slip length was expressed as a function of gas fraction, microchannel constriction, and Reynolds number for Newtonian liquids. Experimental research involving pressure-driven flow through SH-textured micro-channels also estimated the effective slip length to confirm the theoretical predictions (Tsai et al. 2009; Ko et al. 2020). Some recent findings show that the slippery properties of SH-textured surfaces are however compromised due to surfactants adsorbed at the air-water interface (Peaudecerf et al. 2017; Li et al. 2020). Despite the significant progress in understanding the slippage properties on $\mathrm{SH}$ surfaces, the studies are mostly for Newtonian liquids, especially water.

On the other hand, only a few investigations are directed towards the slippage of non-Newtonian liquids on the SH-textured surfaces. A recent review about the slippage of non-Newtonian liquids on randomly and periodically textured surfaces described the wall slip phenomena in detail (Malkin and Patlazhan 2018). Haase et al. (2017) investigated the behaviour of shear-thinning liquids on $\mathrm{SH}$ ribs arranged normal to the flow direction using both numerical simulations and experiments. Compared to water, the shear-thinning liquids exhibited an apparent slip length of more than three times. Micro-particle image velocimetry-based measurements confirmed their numerical results. In another research involving numerical simulations, Patlazhan et al. (Patlazhan and Vagner 2017) studied a shear flow of shear-thinning liquids over ribs arranged parallel and perpendicular to the flow direction. They showed that the apparent slip length associated with shear-thinning liquids is considerably larger than those with Newtonian liquids. Furthermore, the apparent slip length was found to be a non-monotonic function of the shear rate. Crowdy et al. (2017) corroborated these findings, especially, the non-monotonic behaviour of the apparent slip length associated with the shear flow over ribs. In another numerical study, Javaherchian and Moosavi (2019) employed phase-field method to investigate the flow behaviour of power-law liquids in micro-channels with ribbed walls. They reported that pressure drop reduction is more when shear-thickening liquids were employed as compared to shear-thinning liquids.

In summary, the main takeaway of the reported investigations is that the apparent slip length is a non-monotonic function of the shear rate. At the same time, all the efforts were undertaken on one-dimensional geometries such as ribs that are arranged either parallel or normal to the flow direction and they are predominantly shear flows. In addition, there is only one investigation that experimentally demonstrated that the apparent slip length increases when shear-thinning liquids were employed as compared to the Newtonian liquids. Therefore, in this work, we have investigated the pressure-driven flow through micro-channels containing both one-dimensional (longitudinal and transverse ribs) and bi-dimensional (posts and holes) SH textures and elucidated the flow behaviour in detail. In particular, the polysaccharide-based shear-thinning liquids are used as model liquids in both numerical simulations and experiments. The aqueous Xanthan Gum liquids were characterised by the Carreau-Yasuda model. In the numerical simulations, we focus our efforts on understanding the flow behaviour and estimating the friction reduction of shear-thinning liquids on $\mathrm{SH}$ textures. We have varied the non-dimensional shear rate or the Carreau number to compare the influence of texture topography, power-law exponent, and microchannel constriction on friction reduction. In the experiments, we have quantified and compared the flow enhancement of the shearthinning liquids in micro-channels with top and bottom walls covered with either posts or ribs, to those with smooth walls. 


\section{Methodology}

\subsection{Shear-thinning liquids}

Three kinds of shear-thinning liquids were prepared by dissolving Xanthan Gum (Merck, Germany) in de-ionised water. The mass concentration of these aqueous Xanthan Gum (XG) solutions is 1,2 and $5 \mathrm{~g} / \mathrm{L}$, which are designated as XG1, XG2 and XG5, respectively. The shear viscosity properties of these liquids were measured using a MCR 301 Anton-Paar rheometer. The viscosity variation with an increase of shear rate for all the XG liquids is shown in Fig. 1a, indicating a shear-thinning behaviour. The effective viscosity $\left(\eta_{\text {eff }}\right)$ as a function of shear rate $(\gamma)$ of the XG liquids is expressed by Carreau model fit, given by Eq. 1:

$\eta_{\mathrm{eff}}=\eta_{i}+\left(\eta_{o}-\eta_{i}\right)\left[1+(\lambda \dot{\gamma})^{2}\right]^{\frac{(n-1)}{2}}$.

Here, $n$ is power-law exponent, $\lambda$ is relaxation time, $\eta_{i}$ and $\eta_{o}$ are the infinite and zero-shear viscosities, respectively. The fitting parameters for each of the XG liquids are listed in Table 1. These shear-thinning liquids were employed in the flow experiments in SH-textured micro-channels in this research.

On the other hand, the flow behaviour and concomitant friction reduction of the shear-thinning liquid on SH-textured surfaces were investigated numerically by employing only the Carreau parameters of the XG5 liquid, for the sake of simplicity. However, to understand the influence of power-law exponent on the flow behaviour through

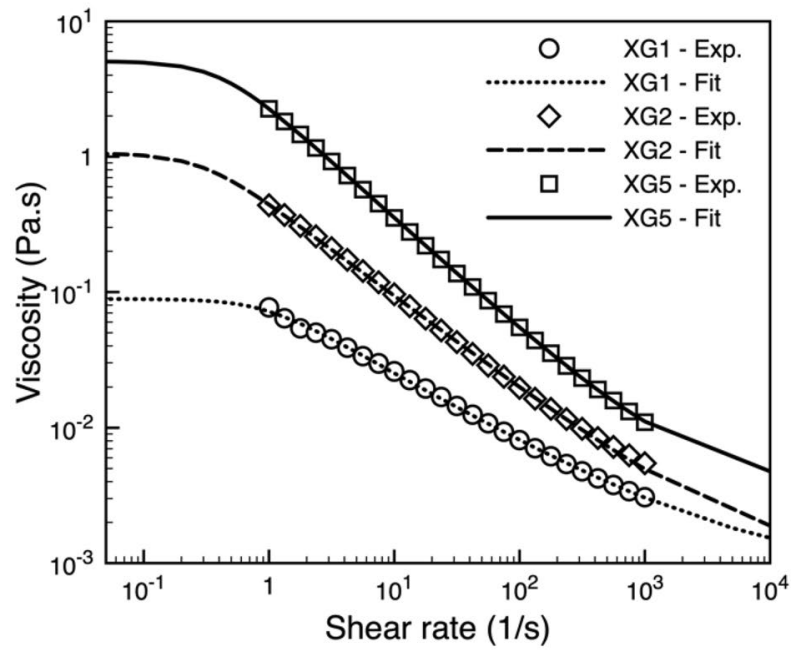

(a)

Fig. 1 a Experimental values and fitted curves for viscosity as a function of shear rate for aqueous Xanthan Gum solutions at different concentrations. b Viscosity as a function of shear rate and power-law
Table 1 Carreau model parameters for the XG liquids of different mass concentrations

\begin{tabular}{llllll}
\hline Designation & $\begin{array}{l}\text { Concentra- } \\
\text { tion }(\mathrm{g} / \mathrm{L})\end{array}$ & $\eta_{i}(\mathrm{~Pa} \mathrm{~s})$ & $\eta \circ(\mathrm{Pa} \mathrm{s})$ & $n$ & $\lambda(\mathrm{s})$ \\
\hline $\mathrm{XG} 1$ & 1 & 0.089 & 0.0009 & 0.472 & 1.12 \\
$\mathrm{XG} 2$ & 2 & 1.059 & 0.0011 & 0.312 & 3.41 \\
$\mathrm{XG5}$ & 5 & 5.078 & 0.0037 & 0.164 & 2.44 \\
\hline
\end{tabular}

numerical simulations, different values of $n$, i.e., $n=0.32$ and 0.48 , were taken from the other XG liquids while keeping the rest of the parameters the same for the XG5 liquid. The effective viscosity as a function of shear rate and powerlaw exponent for the XG5 liquid is shown in Fig. 1b.

\subsection{Numerical details}

To understand the flow behaviour in SH-textured microchannels, we examine four types of SH textures, namely longitudinal ribs, transverse ribs, posts, and holes, as shown in Fig. 2a. Furthermore, we considered sufficiently wide micro-channels for both numerical and experimental investigations. Therefore, the computational domain reduces to a unit cell containing a single $\mathrm{SH}$ texture as shown in Fig. 2b. The liquid flows in an SH-textured microchannel with a height of $2 H$. The unit cell has a size of $L$ with the $\mathrm{SH}$ texture having a characteristic dimension of $s$. Furthermore, the pressure-driven flow is considered to be fully developed and the liquid flows in the $x$-direction. Therefore, due to the periodically repeating flow field in the $x$-direction, the inlet

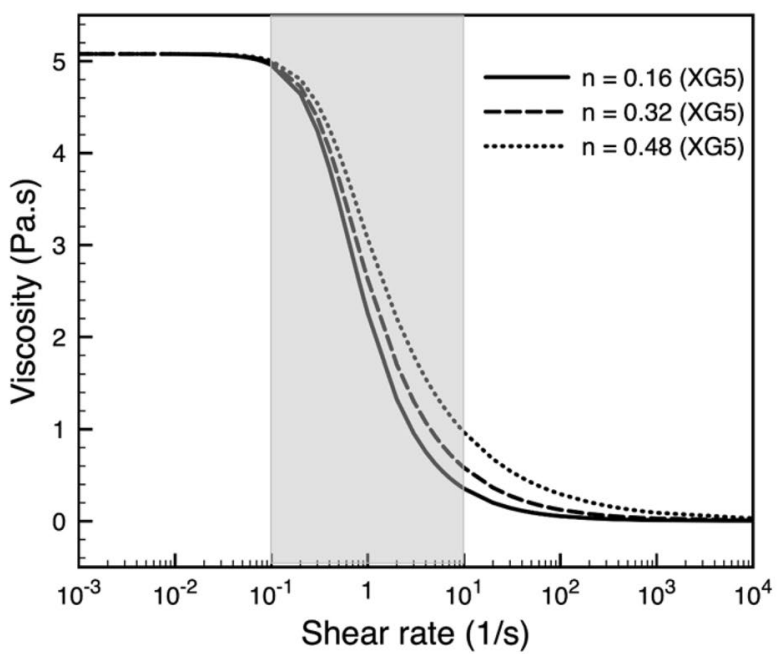

(b)

index ( $n$ ) for an aqueous Xanthan Gum solution with concentration of $5 \mathrm{~g} / \mathrm{L}$. The range of shear rates investigated in this research is shown in shaded area 


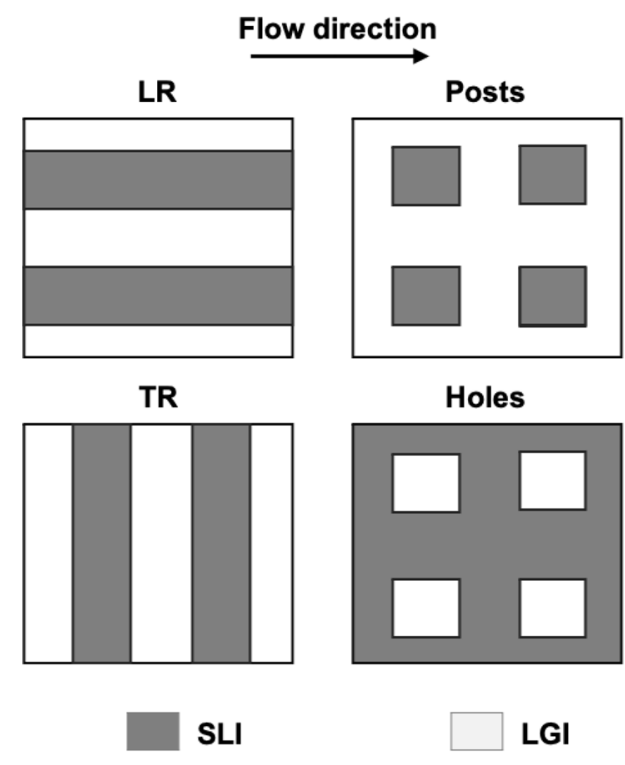

(a)

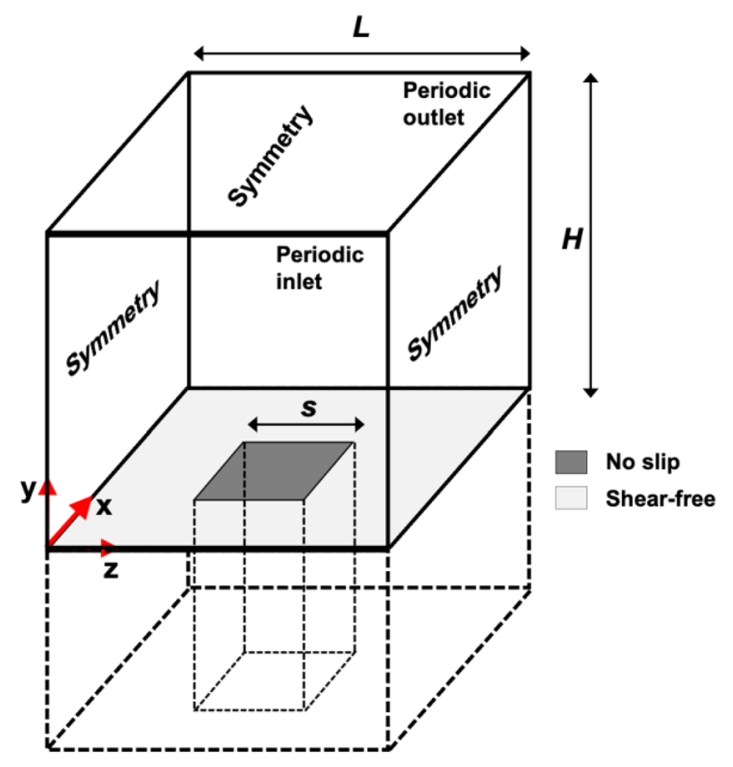

(b)

Fig. 2 a Different surface textures investigated in this work. SLI Solid-liquid interface, LGI Liquid-gas interface. b Computational module containing a single post and boundary conditions are shown

and outlet of the computational domain are designated as the periodic boundaries. In addition, since the microchannel is sufficiently wide ( $z$-direction) exhibiting a $2 \mathrm{D}$ flow condition, the sidewalls of the computational domain are considered to be symmetric boundaries. To further reduce the computational time, half channel height is considered ( $y$-direction) by designating the top wall of the domain to be a symmetric boundary. All the simulations involving solution of the mass and momentum equations were performed in the Ansys Fluent 2020R1 framework.

When the liquid flows over the SH-textured surface, a liquid-gas interface (LGI) forms between the protrusions. The LGI is assumed to be flat and shear-free in this research. This indicates that (1) the deformation of the liquid-gas interface is negligible and (2) the underlying gas layer imparts negligible friction to the liquid flow. Based on the average velocities $(U)$ of the liquid attained in the micro-channels, the capillary number $(\mathrm{Ca}=\mu U / \sigma$, where $\mu$ is the viscosity of the liquid and $\sigma$ is the surface tension of the liquid-gas interface) was estimated. Since the $\mathrm{Ca}$ is a ratio of viscous forces to the surface tension forces, the magnitude of the $\mathrm{Ca}$, therefore, indicates the influence of surface tension on the deformation of the liquid-gas interface. The Xanthan Gum liquids have surface tension and viscosities in the range of 50-72 mN/m (Brunchi et al. 2016) and 0.0037-5.07 Pa.s (see Fig. 1b), respectively. Based on these values, the capillary numbers are evaluated, and our estimates show that $\mathrm{Ca} \ll 1$, which indicates that the surface tension forces are sufficiently high to resist the interface from deformation. At the same time, the shear stress at the liquid-gas interfaces scales as $\tau_{\mathrm{g}^{-} 1} \sim \eta_{\mathrm{g}} / \eta_{\mathrm{l}}$, where $\eta_{\mathrm{g}}$ and $\eta_{\mathrm{l}}$ are the viscosities of the gas and liquid, respectively. Consequently, the magnitude of $\tau_{\mathrm{g}^{-} 1}$ varies from $10^{-3}$ to $10^{-6}$. Such low magnitudes of the interfacial shear stress allowed us to impose the shear-free condition at the liquid-gas interface. The shear-free LGI assumption was previously used for the flow of water in $\mathrm{SH}-$ textured micro-channels (Sharma et al. 2020; Cowley et al. 2016). Although the two-phase models, such as coupled liquid-gas models (Sharma et al. 2019; Maynes et al. 2007) and surface tension-based models (Gaddam et al. 2015; Liu et al. 2021), would improve the accuracy of predictive flow modelling, such models are computationally expensive. Therefore, a computationally fast single-phase model with reasonably valid assumptions aids in investigating the influence of a wide range of parameters on flow enhancement.

The solid-liquid interface (SLI) on the top wall of the $\mathrm{SH}$ texture is assumed to obey the no-slip boundary condition. The pressure-driven flow was initiated by specifying the pressure gradient $(\Delta P / L)$ at the periodic boundaries of the computational module. Since the abrupt changes in viscosity and velocity gradients are expected near the boundary of where LGI and SLI meet, the region near the wall is well-refined. A grid independence test was carried out to find optimal cell size for the computational domain. A maximum number of cells in the computational domain after the refinement range vary anywhere between 0.5 and 1.1 million in the simulations. 
The key non-dimensional parameters pertaining to the microchannel and SH texture geometry are (a) gas fraction, which is defined as the ratio of the area of the LGI to the area of the SLI+LGI and (b) microchannel constriction ratio $(\mathrm{HL}=H / L)$, which is defined as the ratio of the half channel height $(H)$ to the unit cell size $(L)$. The applied shear rate (or pressure gradient) was represented by the non-dimensional Carreau number $(\mathrm{Cu})$, which is a ratio of the characteristic shear rate to the transitional shear rate. Here, the characteristic shear rate is a ratio of the average velocity $(U)$ across the microchannel to the half channel height. At the same time, the transitional shear rate is the shear rate where transition from the Newtonian viscosity in the plateau (see Fig. 1b) to the power-law region occurs. For the XG5 liquid, this transitional shear rate is appeared to be $0.1 \mathrm{~s}^{-1}$ as can be seen from Fig. 1b. The Fanning friction factor, which is expressed as $f=2 \Delta P D_{h} / L \rho U^{2}($ Lemos 2012) was calculated at different Carreau numbers. Here, the $\rho$ is the density of the XG liquid and $D_{h}(=4 H)$ is the hydraulic diameter of the microchannel. Subsequently, the friction factor ratio (FFR), a ratio of friction factor in an SH-textured microchannel to the smooth microchannel, was estimated. It should be noted that the thickness of the gas layer is not considered in this research. It has been shown that the frictional characteristics do not get affected when the aspect ratio of the surface textures is greater than unity (Sharma et al. 2019). Therefore, the single-phase model considered here can accurately predict the friction factor when the thickness of the gas layer is more than the size of the texture.

\subsection{Experimental details}

\subsubsection{Fabrication of super-hydrophobic textured surfaces}

To assess the flow enhancement of XG liquids in SH-textured micro-channels, surfaces containing an array posts and ribs are fabricated by a femtosecond laser micromachining workstation (LASEA LS5, Belgium) on stainless steel (SS). The femtosecond laser has a nominal wavelength of $1032 \mathrm{~nm}$ and a pulse duration of $310 \mathrm{fs}$. The beam was steered through a telecentric focusing lens and scanned at a speed of $1000 \mathrm{~mm} / \mathrm{s}$ using a grid scanning strategy to create posts. The scanning electron microscope (SEM, EOL JCM600) images of the posts and ribs are shown in Fig. 3a. They are further analysed through a focus variation microscope (Alicona G5) to obtain their dimensions. The 3D height map of the surface with posts together with their profile are shown in Fig. 3b. The distance/space between the posts is $75 \mu \mathrm{m}$ and have a size of about $29 \mu \mathrm{m}$, which corresponds to a gas fraction of $\sim 85 \%$. While the spacing between the ribs is $100 \mu \mathrm{m}$ with a size of about $15 \mu \mathrm{m}$, providing a gas fraction of $\sim 85 \%$ on the surface. The side walls of the posts and ribs are in turn covered by the so-called laser-induced periodic

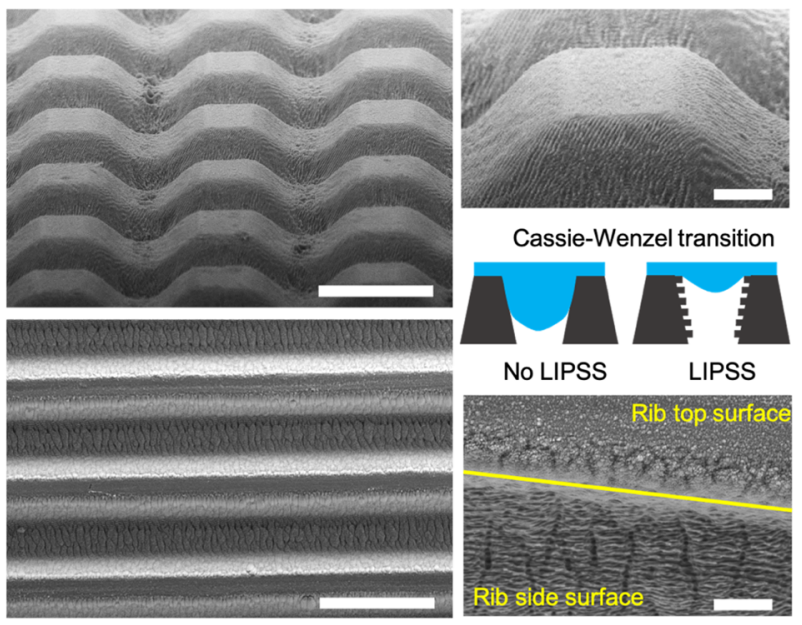

(a)

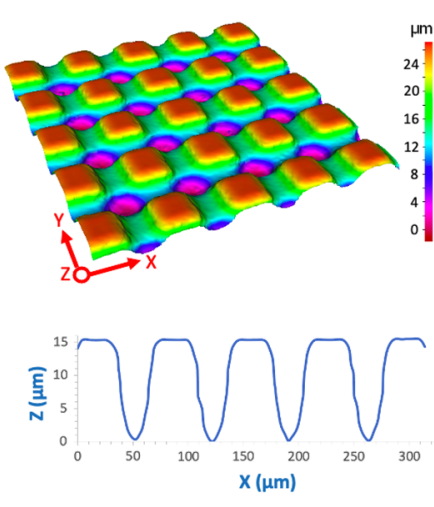

(b)

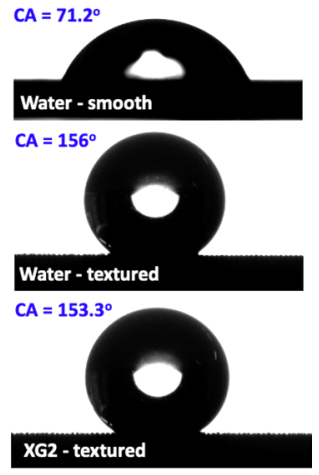

(c)
Fig. 3 a An SEM micrograph of posts (scale bar $50 \mu \mathrm{m}$ ) and ribs (scale bar $100 \mu \mathrm{m}$ ). A magnified view of single posts and ribs showing LIPSS on side walls (scale bar $10 \mu \mathrm{m}$ ). An illustration showing Cassie-Wenzel transition between consecutive posts without and with LIPSS. b A 3D profilometer image and height profile of the posts are shown. c Micrographs showing contact angle on smooth and SH-textured surfaces

surface structures (LIPSS). The LIPSS are nanoscale ripples with a periodicity of 800-900 $\mathrm{nm}$ and depth of $100-200 \mathrm{~nm}$, as confirmed by our previous studies (Gaddam et al. 2021; Siddiquie et al. 2020). Such nanoscale structures on top of microscale features provide an additional energy barrier for wetting and are expected to resist the Cassie-Wenzel transition on SH-textured surfaces (Gaddam et al. 2021; Wu et al. 2017) as shown in Fig. 3a. In addition, the trapezoidal shape of the posts also helps in maintaining the Cassie-Baxter state (Huang et al. 2021).

The textured surfaces are further functionalised by applying Trichloro $(1 \mathrm{H}, 1 \mathrm{H}, 2 \mathrm{H}, 2 \mathrm{H}$-perfluorooctyl) silane (Merck, Germany) to impart the super-hydrophobicity. The contact angle on the smooth and SH-textured SS surfaces was measured using a goniometer (OCA 15EC, Data Physics GmbH, Germany). While the smooth surface exhibited a contact 
angle $(\mathrm{CA})$ of $71.2^{\circ} \pm 2.9$ with water, the contact angle on the $\mathrm{SH}$-textured surfaces was measured to be more than $150^{\circ}$ both with water and XG liquids (see Fig. 3c).

\subsubsection{Fabrication of micro-channels}

The smooth and SH-textured micro-channels were prepared on the same surface with the sticker technique (Kojić et al. 2020). Briefly, an SS sheet was cut into two pieces of $25 \mathrm{~mm} \times 100 \mathrm{~mm}$ size. In one-half of each piece the $\mathrm{SH}$ textures were fabricated by femtosecond laser machining and silane functionalisation. Next, a slot of $2 \mathrm{~mm} \times 70 \mathrm{~mm}$ was machined on a two-side adhesive plastic tape and bonded onto the SS sheets as shown in Fig. 4 to complete the microchannels. Therefore, the top and bottom walls of the SHtextured microchannels were decorated with either posts or ribs. The ribs were machined normal to the flow direction; thus, the configuration is transverse ribs.

The microchannels with two heights were investigated using tapes with a thickness of $90 \mu \mathrm{m}$ (Tesa 64621) and $200 \mu \mathrm{m}$ (3 M 9088). The XG liquids (XG1, XG2 and XG5) were fed through the microchannels with the same inlet using a syringe pump (Legato 110, KD Scientific) as shown in Fig. 4. The inlet pressure was monitored through a pressure gauge (0-4 bar, LEO-Record, Keller, Germany) to ascertain the applied pressure is less than the critical burst pressure required to cause the Cassie-Wenzel transition, to maintain the Cassie-Baxter state throughout the experimental domain. The critical burst pressure for posts and ribs are estimated using the expressions provided elsewhere (Lobaton and Salamon 2007). The volume flow rate from smooth and SH-textured microchannels was estimated by measuring the mass of the liquid collected from the outlets using a precision weighing balance. It is well-known that the properties of some polymers degrade with time of operation. However, the aqueous XG solutions tend to retain their viscosities for up to $330 \mathrm{~h}$ (Zhong et al. 2013). Since the typical length of each experiment is only $0.5-2 \mathrm{~h}$, depending on the flow rate in our work, the XG solutions are expected to be stable during the course of the flow testing.

\section{Flow friction with generic surface textures in micro-channels}

\subsection{Friction factor behaviour of Newtonian and shear-thinning liquids}

After the validation of the numerical set-up (see the supplementary material), a pressure-driven flow of shear-thinning liquid (XG5) through smooth microchannels and microchannels containing ribs arranged normal to the flow direction was simulated to understand the behavior of friction factor. In particular, the microchannels with the constriction ratio of unity (or a height of $200 \mu \mathrm{m}$ ) containing transverse ribs at gas fractions of $50 \%$ and $90 \%$ are considered here. A pressure gradient corresponding to $3 \times 10^{-4} \mathrm{~s}^{-1}<\gamma<3 \times 10^{4} \mathrm{~s}^{-1}$ was applied across the smooth and textured microchannels to estimate the friction factor. Figure 5a shows the friction factor as a function of shear rate. The friction factor for the flow of Newtonian liquid in smooth and textured microchannels is also shown for comparison. Here, the viscosity of the Newtonian liquid corresponds to the upper plateau of the shear-thinning liquid, which is 5.07 (see Fig. 1b).

It is apparent from Fig. 1b that the shear-thinning liquid exhibits a Newtonian behavior for $\gamma<10^{-2} \mathrm{~s}^{-1}$ and $\gamma>10^{2} \mathrm{~s}^{-1}$. The Reynolds number $\left(\operatorname{Re}=\rho U D_{h} / \eta_{\text {eff }}\right)$ calculated based on the average velocity and resulting effective viscosity for a smooth channel corresponding to the above range of shear rates is $3 \times 10^{-9}<\operatorname{Re}<20$. Consequently, the product of friction factor and Reynolds number $(f \mathrm{Re})$ was calculated to be 96 in the Newtonian regime, i.e., $\gamma<10^{-2} \mathrm{~s}^{-1}$ (or $\operatorname{Re}<1 \times 10^{-9}$ ), which is also well in agreement with the classical Hagen-Poiseuille flow. Therefore, as can be seen from Fig. 5a, the slope of the friction factor curve for shear-thinning liquid is linear for $\gamma<10^{-2} \mathrm{~s}^{-1}$ (Newtonian

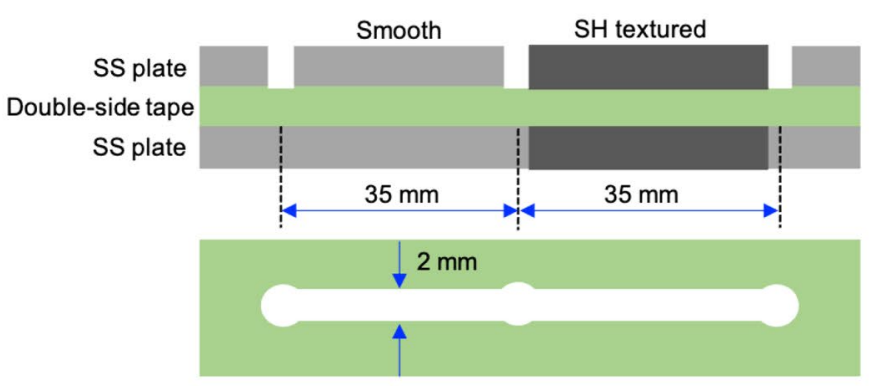

(a)

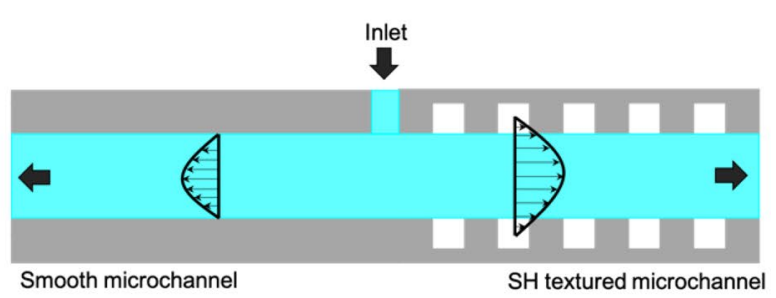

(b)

Fig. 4 a An exploded view of the microchannel configuration employed for the experiments. b An illustration of slip flow in smooth and SHtextured microchannels is also shown 


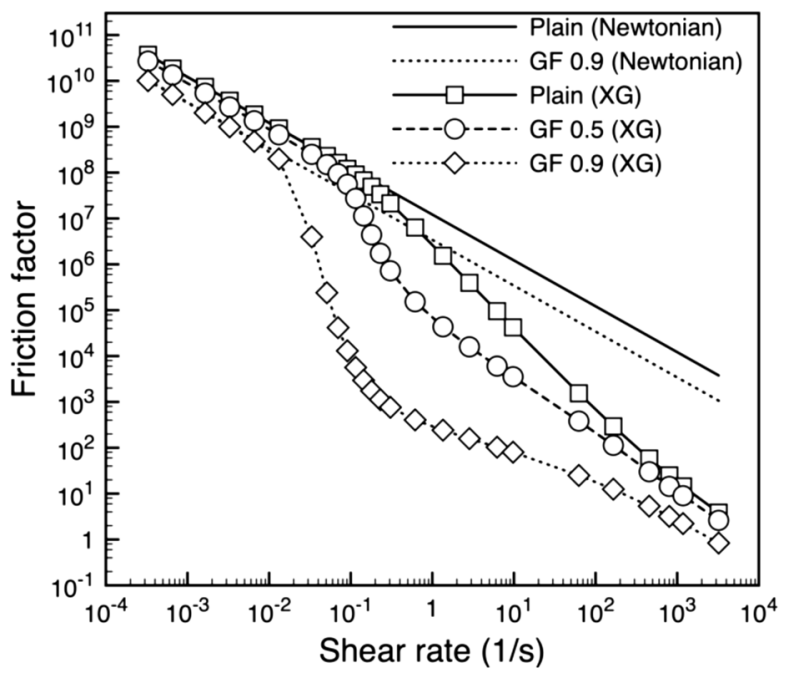

(a)

Fig. 5 a Friction factor as a function of shear rate for a microchannel with plain surfaces and textured microchannels with a gas fraction of $50 \%$ and $90 \%$. b Friction factor ratio as a function of shear rate

regime) for the smooth microchannel and aligning well with the Newtonian one. At the same time, the value of $f \operatorname{Re}$ is constant when the inertial effects are not predominant for the Newtonian liquids in the SH-textured microchannels as confirmed by the previous studies (Sharma et al. 2020; Brunchi et al. 2016; Cowley et al. 2016; Samaha et al. 2011). Consequently, the slope of the friction factor curve is also linear for the SH-textured microchannels in the Newtonian regime. Finally, when the applied shear rates are again in the lower Newtonian plateau i.e., $\gamma>10^{2} \mathrm{~s}^{-1}$, the slope of the friction factor curve tending towards linearity for both the smooth and SH-textured microchannels as shown in Fig. 5a. This behaviour of the friction factor in smooth channels as a function of shear rate in the entire regime for XG liquids is also similar to what was observed in another investigation (Shende et al. 2021).

On the other hand, the friction factor showed a distinct non-linear behavior in the shear-thinning regime $\left(10^{-2} \mathrm{~s}^{-1}<\gamma<10^{2} \mathrm{~s}^{-1}\right)$ for flow past transverse ribs at different gas fractions. To assess the flow friction behavior of shear-thinning liquid in SH-textured microchannels, the friction factor ratio is plotted as a function of shear rate in Fig. $5 \mathrm{~b}$ and compared with Newtonian liquid. As can be seen, the FFR is constant at all the shear rates for Newtonian liquids flow past SH-textured microchannels with different gas fractions. However, FFR remained constant for $\gamma<10^{-2} \mathrm{~s}^{-1}$ for the shear-thinning liquid in the Newtonian regime, followed by a clear departure at $\gamma>10^{-2} \mathrm{~s}^{-1}$ for the SH-textured microchannels. In the shear-thinning regime $\left(10^{-2} \mathrm{~s}^{-1}<\gamma<10^{2} \mathrm{~s}^{-1}\right)$, the minimum of FFR was noticed

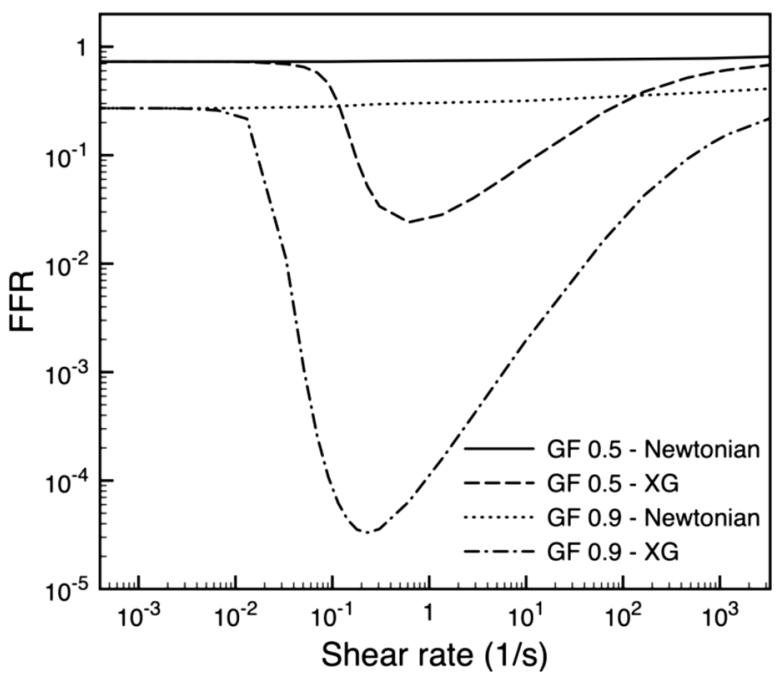

(b)

for Newtonian and shear-thinning (XG) liquids fed through textured microchannels with a gas fraction of $50 \%$ and $90 \%$

at a shear rate of $\sim 0.2 \mathrm{~s}^{-1}$ and $\sim 0.6 \mathrm{~s}^{-1}$ for microchannels with a gas fraction of $90 \%$ and $50 \%$, respectively. Once the Newtonian regime is established at the high shear rates $\left(\gamma>10^{3} \mathrm{~s}^{-1}\right)$, the FFR is observed to plateau towards constant FFR curves.

\subsection{Viscous dissipation with Newtonian and shear-thinning liquids}

To further validate how the minima of the friction factor ratio occur in the shear-thinning regime $\left(10^{-2} \mathrm{~s}^{-1}<\gamma<10^{2} \mathrm{~s}^{-1}\right)$, the viscous dissipation rate is estimated in the smooth and textured microchannels for a flow of water and shear-thinning liquid. In particular, the viscous dissipation rate per unit volume $(\varphi)$ for a $2 \mathrm{D}$ flow is calculated numerically as (Winter 1987):

$\varphi=2 \eta_{\mathrm{eff}}\left\{\left[\left(\frac{\mathrm{d} u}{\mathrm{~d} x}\right)^{2}+\left(\frac{\mathrm{d} v}{\mathrm{~d} y}\right)^{2}\right]+\frac{1}{2}\left[\frac{\mathrm{d} u}{\mathrm{~d} y}+\frac{\mathrm{d} v}{\mathrm{~d} x}\right]^{2}\right\}$.

Here, $u$ and $v$ are the velocity components in the $x$ and $y$ directions. The velocity and length quantities are normalized by the average velocity $(U)$ and half channel height $(H)$ to make the viscous dissipation rate a dimensionless quantity. In addition, we considered microchannels with a constriction ratio of unity and contained transverse ribs at a gas fraction of $90 \%$, and the shear rates are varied from 0.01 to $10 \mathrm{~s}^{-1}$ to calculate the non-dimensional viscous dissipation rate $(\phi)$. Then, the viscous dissipation ratio ( $\phi$ ratio), which is a ratio of the viscous dissipation rate in the $\mathrm{SH}$-textured 


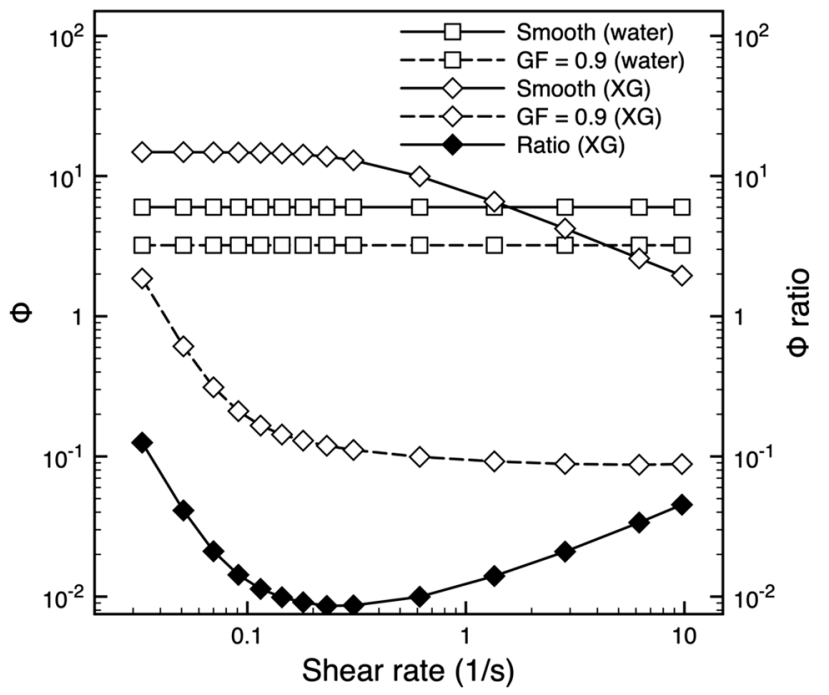

Fig. 6 Viscous dissipation rate (open symbols) as a function of shear rate for water and shear-thinning liquid flowing in smooth and textured microchannels. The viscous dissipation ratio (filled symbols) in $\mathrm{SH}$-textured and smooth microchannels for a shear-thinning liquid is also shown. Here, the $\mathrm{GF}=0.9$ and $\mathrm{HL}=1$

microchannel to the smooth channel is calculated for water and shear-thinning liquids. First, the dimensionless viscous dissipation rate is estimated for a flow of water in a smooth channel. As shown in Fig. 6, irrespective of the shear rate, the viscous dissipation for water in a smooth channel with a constriction ratio of unity takes a value of 6 , which agrees with another investigation (Haase et al. 2016). At the same time, the viscous dissipation rate reduced to a value of 3.21 at all the shear rates for a flow of water in textured microchannels. Therefore, the viscous dissipation ratio for the Newtonian liquids is constant $(\phi$ ratio $=0.53)$. In the case of shear-thinning liquid, the viscous dissipation rate in the smooth channel reduced from $14.8\left(\gamma=0.03 \mathrm{~s}^{-1}\right)$ to 1.9 $\left(\gamma=9.7 \mathrm{~s}^{-1}\right)$. Similarly, the viscous dissipation rate in the SH-textured microchannels also decreased with an increase in the shear rate. While the reduction in viscous dissipation rate in smooth microchannels is gradual until a shear rate of about $0.2 \mathrm{~s}^{-1}$, it decreased rapidly in the textured microchannels. The ratio of viscous dissipation rate for the shear-thinning liquid is minimized at a shear rate of $0.23 \mathrm{~s}^{-1}$ as shown in the figure.

\subsection{Influence of Carreau number}

Since the shear-thinning behaviour is predominantly observed in the range $10^{-2}<\gamma<10^{2}$, the numerical simulations were performed for a flow past $\mathrm{SH}$ textures, such as posts, holes, longitudinal and transverse ribs, in microchannels by applied the pressure gradients corresponding to the shear rates in that regime. In particular, the Carreau number $(\mathrm{Cu})$ was varied between 0.03 and 97.5 , while the microchannel constriction ratios (HL) from 0.5 to 2 and the power-law exponents from 0.16 to 0.48 were investigated. The microchannel constriction ratios $0.5,1$ and 2 correspond to channel heights of $100 \mu \mathrm{m}, 200 \mu \mathrm{m}$ and $500 \mu \mathrm{m}$, respectively. The gas fraction of SH textures in the microchannels was varied from 0.5 and 0.9 . Figure 7 shows the friction factor ratio as a function of the Carreau number for all SH textures in microchannels with a constriction ratio of unity and a gas fraction of $90 \%$. The friction factor ratio was observed to rapidly decrease until $\mathrm{Cu} \sim 2.3$ for all the $\mathrm{SH}$ textures before a gradual increasing trend. Similar to the behaviour of the Newtonian liquids (Cheng et al. 2009), the longitudinal ribs and posts outperformed all the SH textures for shear-thinning liquids, too, when compared to the transverse ribs and holes. This is because liquids undergo periodic acceleration-deceleration cycles for a flow past holes and transverse ribs on the slip and non-slip interfaces that are normal to the flow direction. This leads to friction losses in such discontinuous $\mathrm{SH}$ texture configurations, whereas such losses are not present in the case of continuous SH textures, such as longitudinal ribs and posts. Such non-intermittent $\mathrm{SH}$ textures not only reduce the friction losses but also help in negating the detrimental effects of contamination at the liquid-gas interfaces (Gaddam et al. 2018).

To understand the flow behaviour of the shear-thinning liquid at the liquid-gas interface, the velocity profiles are plotted in Fig. 8. Here, the interfacial velocities of the SH-textured microchannels are normalized by the average velocity $(U)$ across the corresponding smooth microchannel. Figure 8 a shows the velocity profiles of

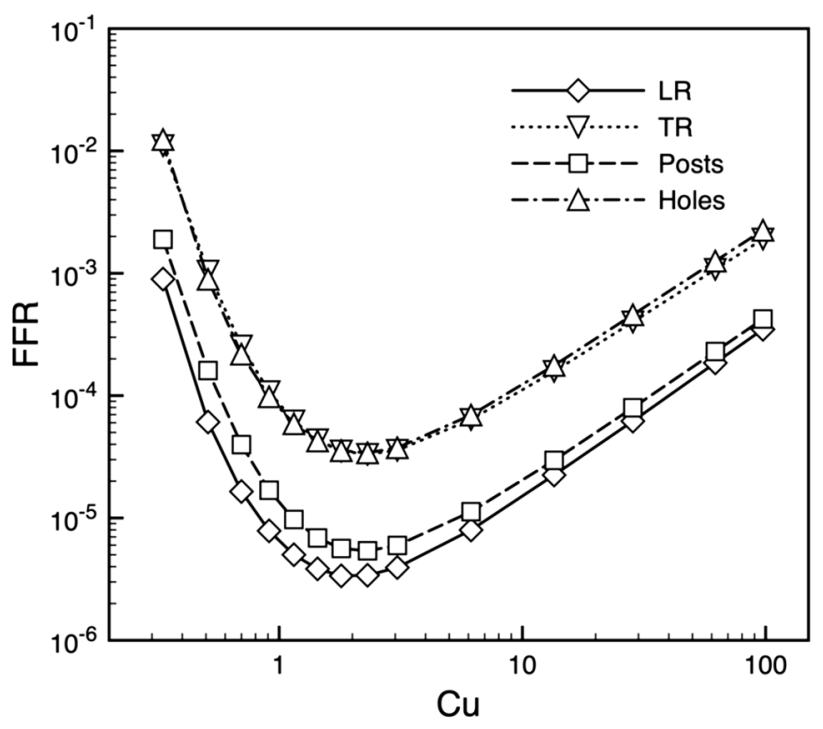

Fig. 7 Friction factor ratio as a function of Carreau number for all the SH surface textures. The textured microchannel constriction ratio here is $\mathrm{HL}=1$ 


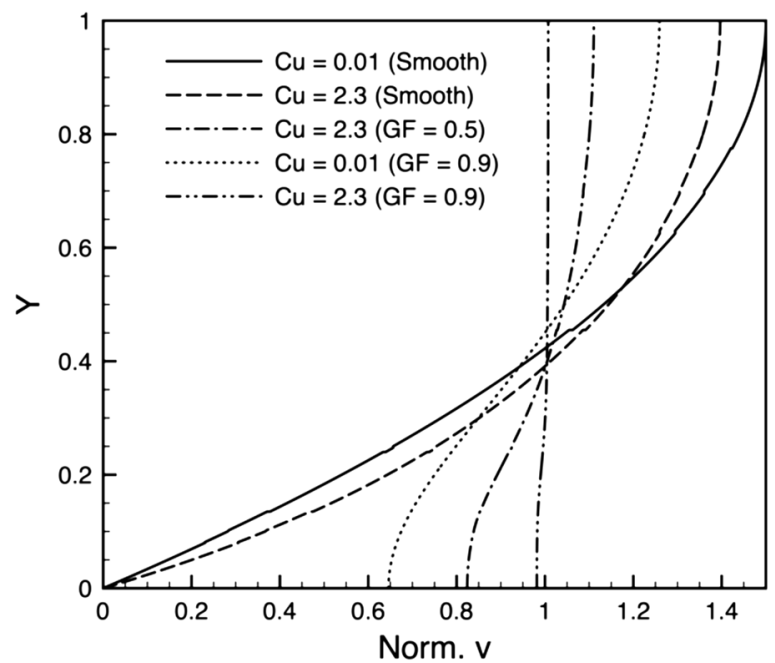

(a)

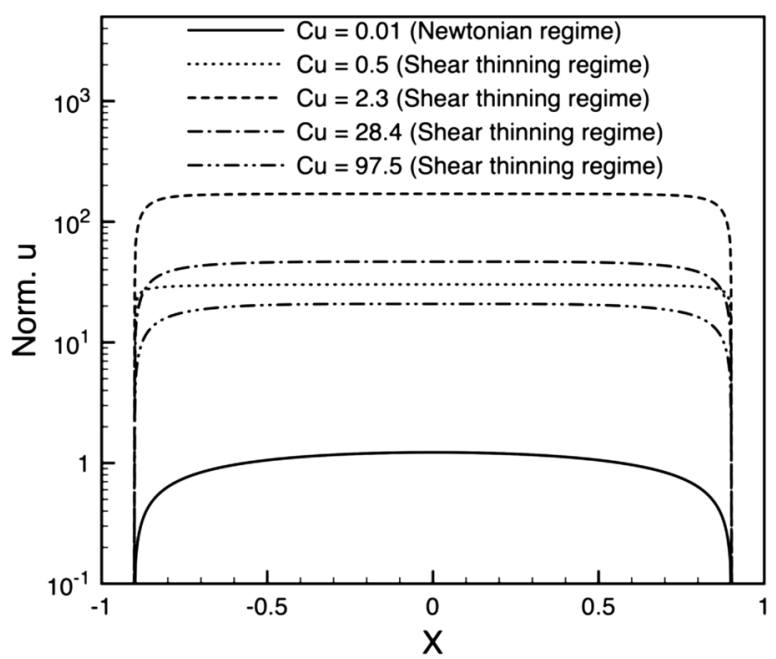

(b)

Fig. 8 a The normalized slip velocity profiles and $\mathbf{b}$ the normalized interfacial velocity profiles for flow past transverse ribs obtained at different Carreau numbers. The microchannel constriction ratio here is $\mathrm{HL}=1$

the shear-thinning liquid at different Carreau numbers in smooth microchannels and microchannels containing transverse ribs arranged at a gas fraction of $50 \%$ and $90 \%$. The velocity profiles for the SH-textured microchannels are extracted at the centre of the liquid-gas interface $(x / L=0.5)$. As can be seen, when the shear rate is in the Newtonian regime $(\mathrm{Cu}=0.01)$, the maximum velocity $\left(U_{\max }\right)$ is $1.5 U$ in a smooth microchannel. Whereas, in the shear-thinning regime where the friction factor minima was observed at $\mathrm{Cu} \sim 2.3, U_{\max }$ is reduced to $\sim 1.3 U$. At the same time, when the gas fraction is $90 \%$, the normalized slip velocity $\left(U_{\text {slip }}\right)$ for $\mathrm{Cu}=2.3$ at the liquid-gas interface was estimated to be $\sim 1.5$ times that of $\mathrm{Cu}=0.01$. This indicates that the slippage at the liquid-gas interface is more in the shear-thinning regime as compared to the Newtonian regime for the same shear-thinning liquid. In addition, as can be seen from the figure, the shear-thinning regime has pronounced slippage even at lower gas fractions (50\%) when compared to the slippage at high gas fractions $(90 \%)$ in the Newtonian regime.

Figure $8 \mathrm{~b}$ shows the normalized interfacial velocity profiles for transverse ribs at a gas fraction of $90 \%$ at different Carreau numbers. In the Newtonian regime $(\mathrm{Cu}=0.01)$, the interfacial velocity profile shows a parabolic behaviour (note that the normalised interfacial velocity is on the log-scale). On the other hand, such behaviour is no longer present in the shear-thinning regime $(\mathrm{Cu}=2.3-97.5)$, where the normalized interfacial velocity increases steeply near the LGI/ SLI boundary and reached a plateau along the length of the interface. Also, the interfacial velocity profile has the highest magnitude at $\mathrm{Cu}=2.3$, where the friction factor minima was observed.

\subsection{Influence of gas fraction}

Next, the influence of gas fraction on the friction factor ratio was investigated. Figure 9a shows the friction factor ratio as a function of gas fraction (50\%-90\%) for all the SH textures when the microchannel constriction ratio is unity. At lower gas fractions (50\%), except for the longitudinal ribs, other SH textures showed similar behaviour. However, with an increase in the gas fraction the posts exhibited a significant decrease in the friction factor ratio. At high gas fractions $(90 \%)$, while the posts and longitudinal ribs resulted in a low friction factor ratio, the holes and transverse ribs underperformed when compared to them. It should be noted that these trends associated with the flow of shear-thinning liquids past the SH textures are similar to the behaviour of Newtonian liquids (Cheng et al. 2009). The effect of the gas fraction on the minima of friction factor ratio in the shearthinning regime is elucidated through Fig. 9b. As can be seen, the friction factor ratio minima is shifted from $\mathrm{Cu} \sim 2.3$ at high gas fractions $(90 \%)$ to $\mathrm{Cu} \sim 6.1$ at low gas fractions $(50 \%)$ for the flow past the posts. A similar behaviour is also observed for the other SH textures.

\subsection{Influence of microchannel constriction ratio}

Another important parameter that influences the flow behaviour in SH-textured microchannels is the constriction ratio. At high constriction ratios, i.e., when the separation between the top and bottom walls is sufficiently large, the flow field generated the slip and no-slip regions on the walls does not interact with each other. However, when the separation between the walls decreases, an interaction of flow field 


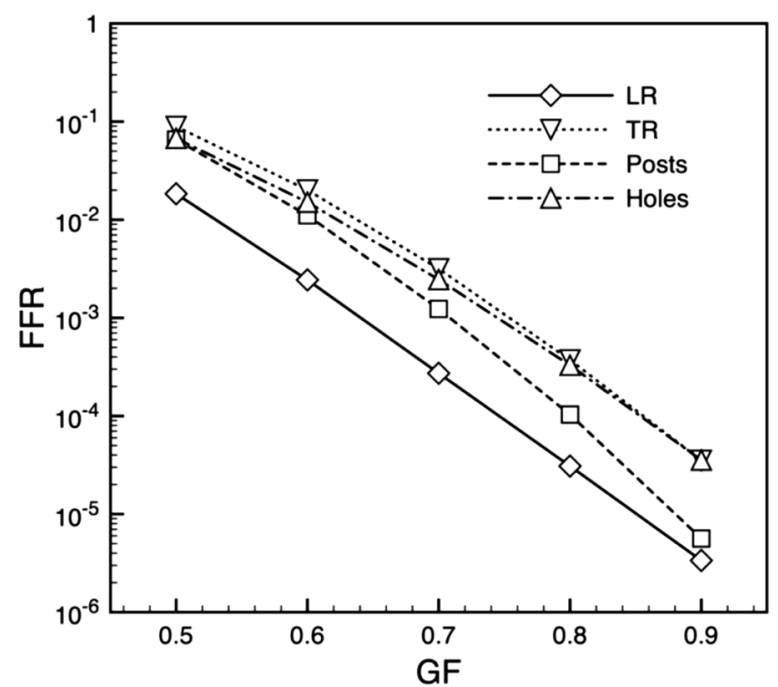

(a)

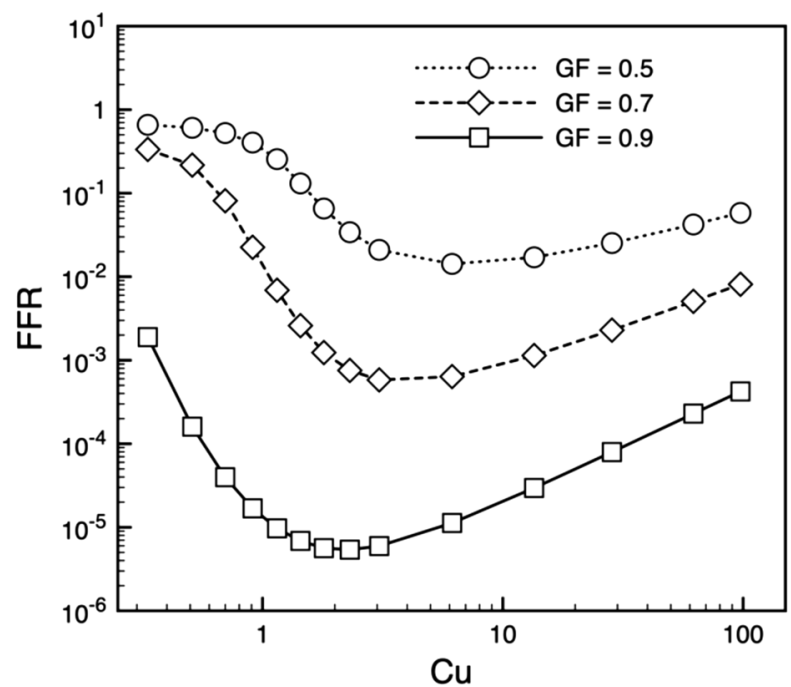

(b)

Fig. 9 Friction factor ratio as function of a gas fraction for all the SH surface textures and $\mathbf{b}$ Carreau number for posts at different gas fractions. Here is $\mathrm{HL}=1$

from the walls could influence the overall flow behaviour and thus flow friction. To assess this, the constriction ratio of the microchannels varied from 0.5 to 2.5 in the simulations.

The friction factor ratio as a function of the Carreau number at different constriction ratios for all the SH textures is shown in Fig. 10. As can be seen, the friction factor ratio decreased by $\sim 70 \%$, when the constriction ratio was decreased from 2.5 to 1 and from 1 to 0.5 . A further decrease in the constriction ratios to 0.25 and 0.1 also led to a similar magnitude of reduction in the friction factor ratio (not shown) for all the SH textures. This indicates that the reduction in the microchannel height for a given period of the $\mathrm{SH}$ texture yields a considerable benefit in terms of slippage for the shear-thinning liquids, which is similar to the Newtonian liquids (Sharma et al. 2019; Kant and Pitchumani 2021). Furthermore, the minima of friction factor ratio is noticed to be at $\mathrm{Cu} \sim 2.3$ for a gas fraction of $90 \%$, irrespective of the microchannel constriction ratio for all the $\mathrm{SH}$ textures. At the same time, the friction factor ratio decreases by an order of magnitude with an increase in the microchannel height by two times as shown in Fig. 10. Therefore, the laminar drag reduction becomes negligible in those channels with a height of tens of millimetres.

At the same time, the presence of slip (LGI) and no-slip (SLI) regions on the walls causes the disturbance in the flow velocity and shear rate field and thus influences the distribution of viscosity near the walls. Figure 11 shows the shear rate and viscosity distribution with an increase in the Carreau number in low constriction ratio $(\mathrm{HL}=0.5)$ microchannels decorated with posts. As can be seen, there exists a stark difference in the shear rate distribution near the slip and no-slip regions when the liquid inertia is low. This also results in a tangible variation in the viscosity distribution at the walls. However, with increased liquid inertia, the sudden jump in the shear rate near the LGI/SLI boundaries vanishes. Consequently, the viscosity distribution near the wall resembles the same pattern as the shear rate as shown in the figure.

The interaction of the flow field due to the separation between the top and bottom walls is represented by the viscosity variation in the microchannels as shown in Fig. 12. Since the local viscosity is a function of the local shear rate inside the domain, the variation of viscosity along the microchannel height is plotted for the microchannels with different constriction ratios. Here the viscosity is normalized by the zero-shear rate viscosity. The viscosity variation normal to the flow direction for a flow past posts in the microchannel constriction ratio of $0.5,1$ and 2.5 is shown in Fig. 12a-c, respectively. As can be seen, when the top and bottom walls are closer to each other $(\mathrm{HL}=0.5)$, the local viscosity showed a dramatic variation even near the half channel height $(y / H=0.9)$. Especially, the wall effect has profoundly influenced the viscosity till $50 \%(y / H=0.5)$ of the half channel height for $\mathrm{HL}=0.5$. At the same time, even though the separation between the top and walls is increased to $\mathrm{HL}=1$, the wall effect is significantly pronounced up to $30 \%(y / H=0.3)$ of the half channel height. Although, the viscosity variation in this case is suppressed after $y / H=0.75$. That means, for the microchannel with a height of $200 \mu \mathrm{m}$, the wall effects are present up to a distance of $75 \mu \mathrm{m}$ from each wall, given the period of the $\mathrm{SH}$ texture is less than $100 \mu \mathrm{m}$. A further increase in the microchannel constriction ratio to 2.5 has led to suppression of wall effects, i.e., the 


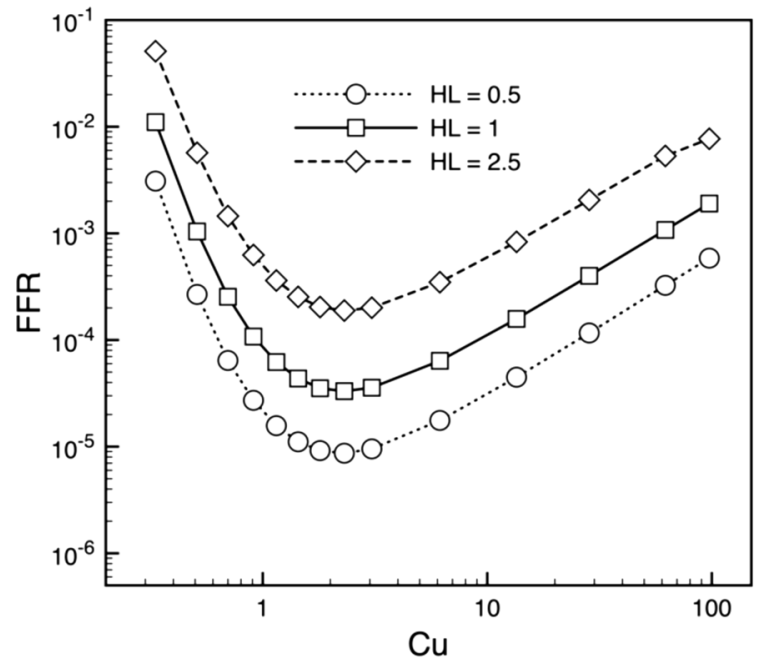

(a)

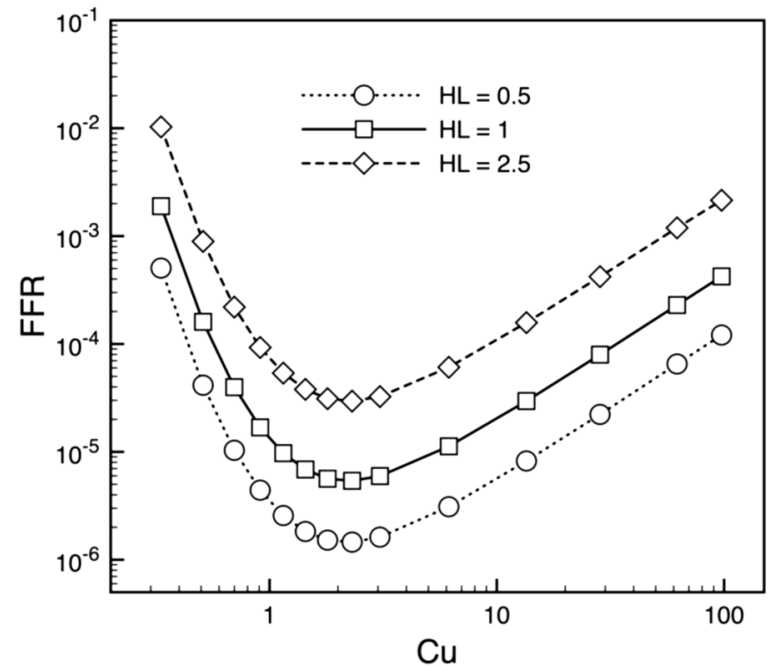

(c)

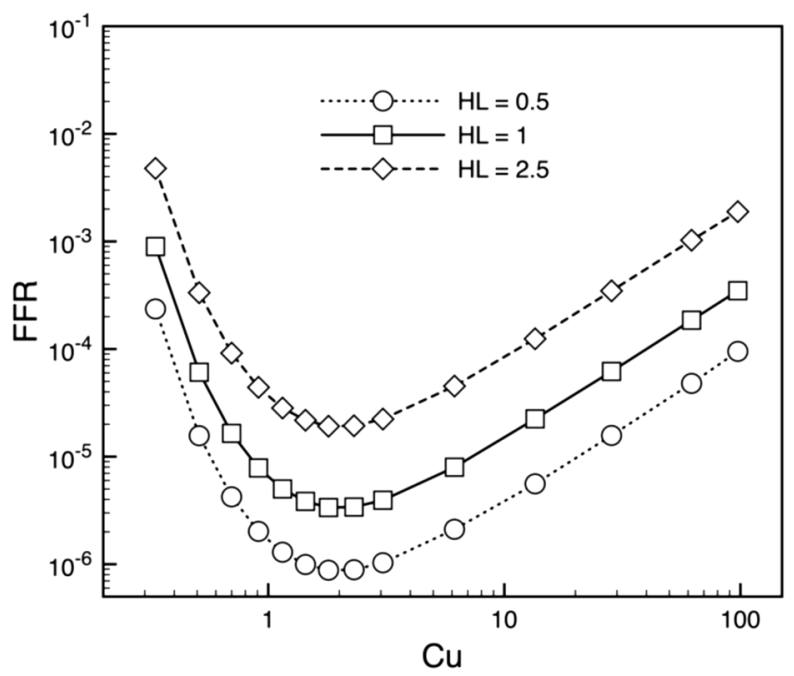

(b)

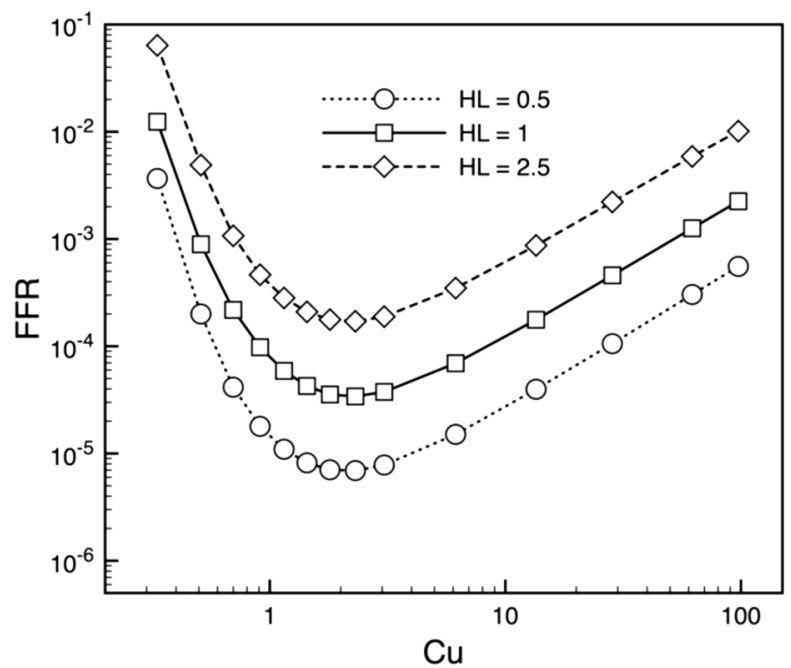

(d)

Fig. 10 Friction factor ratio as a function of Carreau number for microchannels with different constrictions ratios when the microchannels are decorated with a transverse ribs, $\mathbf{b}$ longitudinal ribs, $\mathbf{c}$ posts and $\mathbf{d}$ holes at a gas fraction of $90 \%$

viscosity variation is noticed up to $20 \%$ of the half channel height. The viscosity contours across a single post in the microchannels with $\mathrm{HL}=0.5,1$ and 2.5 shown in Fig. 12d, elucidates the viscosity variation.

\subsection{Influence of power-law exponent}

Since the concentration of XG dictates the shear-thinning behaviour of the XG liquids as can be seen in Table 1, here, we attempted to investigate the strongly and weakly shearthinning liquids on the flow behaviour and friction factor ratio. The strong or weak nature of the shear-thinning liquids can be described by viscosity ratio $(\alpha$, a ratio of the infinite shear viscosity and zero-shear viscosity) and power-law exponent $(n)$. For instance, $\alpha$ takes a value of 1 for the water, whereas for the XG liquids $\alpha<1$. At the same time, $n=1$ for the water, while $n<1$ for the XG liquids. The lower the value of either $\alpha$ or $n$, the stronger the shear-thinning nature. Here, for the sake of simplicity, we kept $\alpha$ constant and $n$ is varied at three levels, i.e., $0.16,0.32$ and 0.48 corresponding to the XG5, XG2 and XG1 liquids. Figure 13a, b shows the friction factor ratio as a function of the Carreau number for a flow past longitudinal ribs and posts, respectively. Here, the microchannel constriction ratio is unity, and the gas fraction is $90 \%$. As can be seen, the weaker the shear-thinning liquid, the more the attenuation of non-linearity of the friction factor ratio curve. Furthermore, the minima of the friction factor ratio is shifting from $\mathrm{Cu} \sim 2.3$ at $n=0.16$ to $\mathrm{Cu} \sim 6.1$ at 


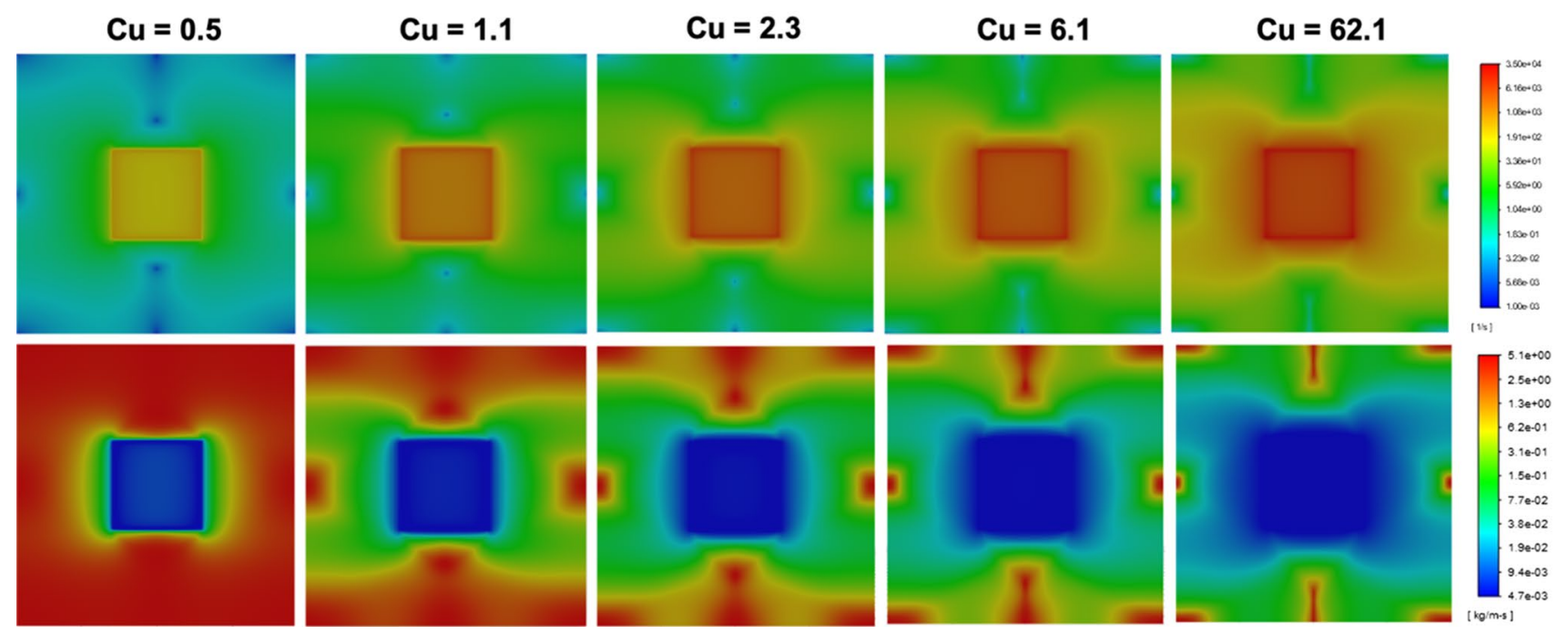

Fig. 11 The shear rate (top panel) and viscosity (bottom panel) distribution on the bottom wall at different Carreau numbers for a flow past single post in a microchannel with $\mathrm{HL}=0.5$. The flow direction is from left to right

$n=0.32$ to $\mathrm{Cu} \sim 13.5$ at $n=0.48$ for all the SH textures. It is apparent that the FFR curve tends towards linearity with as $n \rightarrow 1$. A similar behaviour was observed for microchannels with different constriction ratios.

The normalized viscosity profiles at the wall along the microchannel height for all the power-law exponents are shown in Fig. 13c. As can be seen, when the weak shearthinning liquids ( $n=0.16)$ are employed, the disturbance in the viscosity near the LGI/SLI boundary is apparent due to the sudden jump in the shear rate. However, disturbance in the viscosity near the LGI/SLI boundary is being vanished as the shear-thinning liquid is becoming stronger. The viscosity contours along the microchannel height normal to the flow direction shown in Fig. 13d is also confirming that the viscosity disturbance is attenuating with an increase in $n$. It is apparent that when $n \rightarrow 1$, the disturbance in the viscosity due to the LGI/SLI boundaries confine to the wall. The interfacial velocities for a single post in a microchannel for Newtonian and shear-thinning liquids shown in Fig. 14a also confirms that the velocity near the LGI/SLI boundaries steeply varying for strongly shear-thinning liquid $(n=0.16)$. Whereas an increase in the $n$ is attenuating the curvature of the velocity profiles near the boundaries. The corresponding shear stress profiles for the Newtonian and shear-thinning liquids shown in Fig. 14b also confirm the same.

\subsection{Influence of relaxation time}

To investigate whether liquid relaxation time $(\lambda)$ has any influence on the friction factor ratio, numerical simulations were performed by varying the $\lambda$ at three levels, i.e., 0.24 , 1.22 and 2.44 of the XG5 liquid by keeping the rest of the parameters same. As can be seen from Fig. 15a, decreasing the relation time from 2.44 to 1.22 has led to a shift in the transitional shear rate from 0.1 to $0.3 \mathrm{~s}^{-1}$, and a further decrease to 0.24 resulted in a transitional shear rate of $1 \mathrm{~s}^{-1}$. Subsequently, the Carreau number is redefined taking these transitional shear rates into the account for numerical simulations. Figure $15 \mathrm{~b}$ shows the friction factor ratio for a flow past posts in a microchannel with $\mathrm{HL}=1$ and $\mathrm{GF}=0.9$. It is apparent that the friction factor ratio remains the same for all the relaxation times considered. That is, the relaxation time shows no influence on the flow behaviour of the shearthinning liquids in the SH-textured micro-channels. It also confirmed that the Carreau number can be considered as a non-dimensional parameter while comparing the flow behaviour on geometrically different rough surfaces and considering shear-thinning liquids with different properties. Finally, empirical correlations were developed for the frication factor ratio as a function of the Carreau number at different gas fractions, power-law exponents, and microchannel constriction ratios (see the supplementary material).

\subsection{Discussion on minima of the friction factor ratio}

As can be seen in previous sections, the friction factor ratio was noticed to be minimized for the shear-thinning liquids at certain shear rates. Some of the previous research also reported that the hydrodynamic drag minimises on the $\mathrm{SH}$ textures when shear-thinning liquids are employed (Haase et al. 2017; Patlazhan and Vagner 2017; Crowdy 2017). It is well-accepted that the reduction in hydrodynamic friction for a flow of shear-thinning liquid is attributed to the depletion layer near the wall having considerably less viscosity than the bulk liquid as shown in Fig. 16a. Consequently, Patlazhan and Vagner (2017) showed that the ratio 


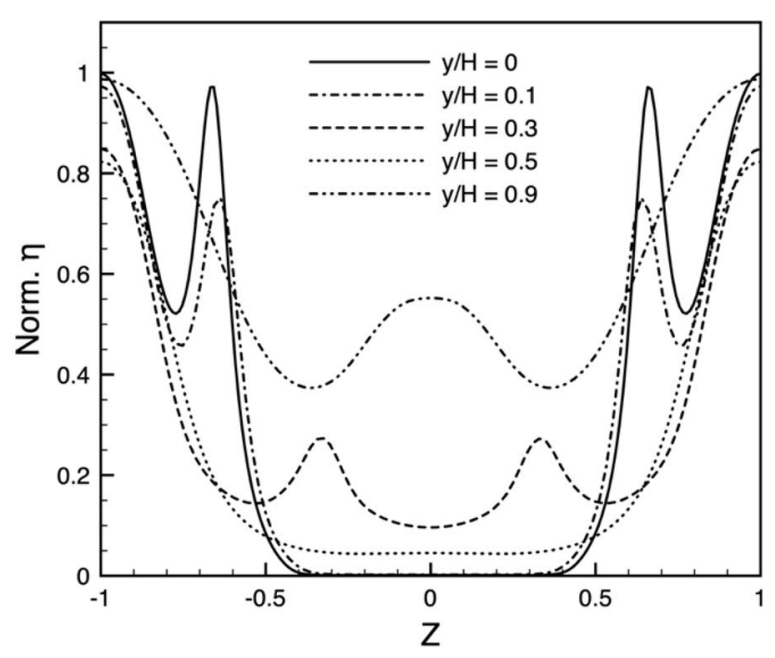

(a)

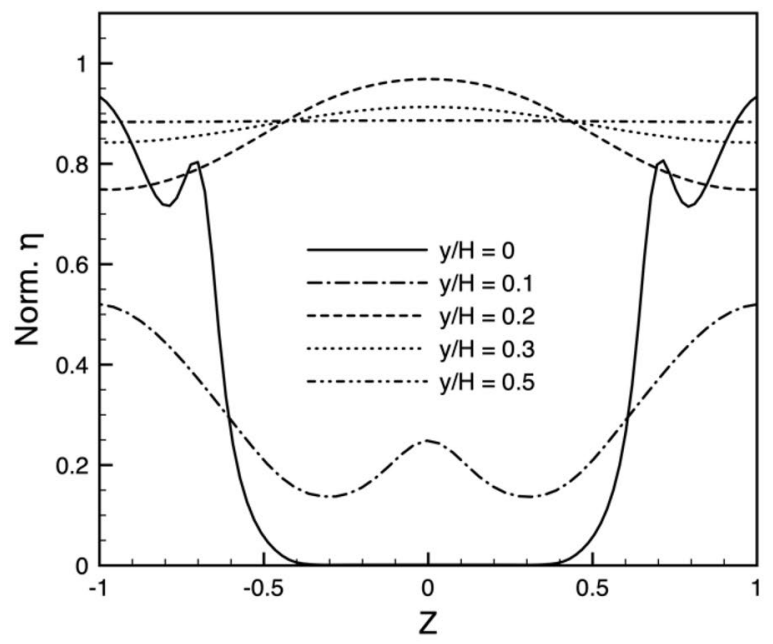

(c)

Fig. 12 The viscosity variation normal to the flow direction at different heights for a post in textured microchannels with a constriction ratio of $\mathbf{a} \mathrm{HL}=0.5, \mathbf{b} \mathrm{HL}=1$ and $\mathbf{c} \mathrm{HL}=2.5$ and at $\mathrm{Cu}=3$. The vis-

of viscosities $\left(\eta\right.$. ratio $=\eta_{d} / \eta_{b}$, where $\eta_{d}$ and $\eta_{b}$ are the viscosities of the depletion and bulk layers, respectively) in the depletion layer to the bulk governs the non-monotonic behavior of the hydrodynamic drag in shear-thinning liquids. However, determination of the thickness of the depletion layer is essential to establish the non-monotonic variation of the $\eta$. ratio, which is not addressed earlier. In addition, it is also noted in the previous work that such non-monotonic variation in $\eta$. ratio is not apparent in flow over high gas fraction SH-textured surfaces due to the shear rate disturbances present in the microchannels (Patlazhan and Vagner 2017). Therefore, here we analysed the behaviour of the depletion layer to understand the non-monotonic variation in the hydrodynamic friction curves.

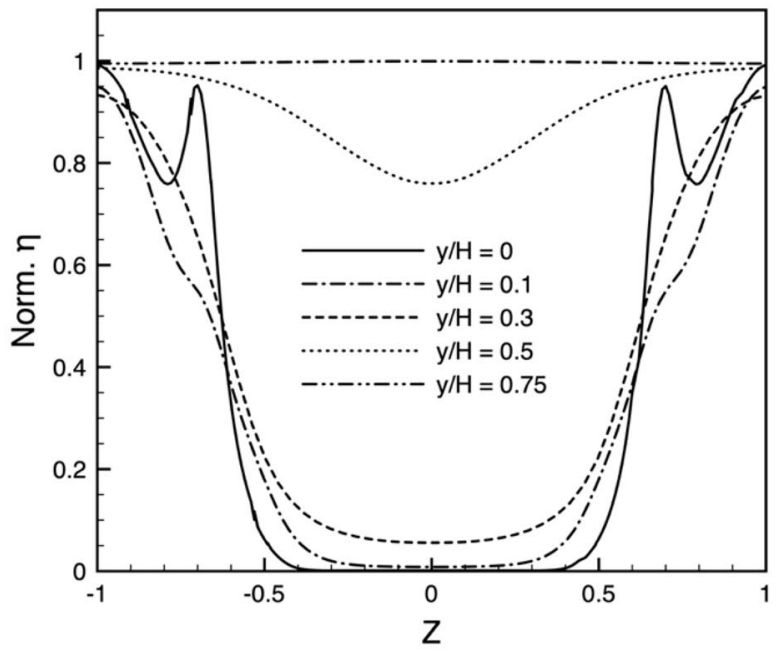

(b)

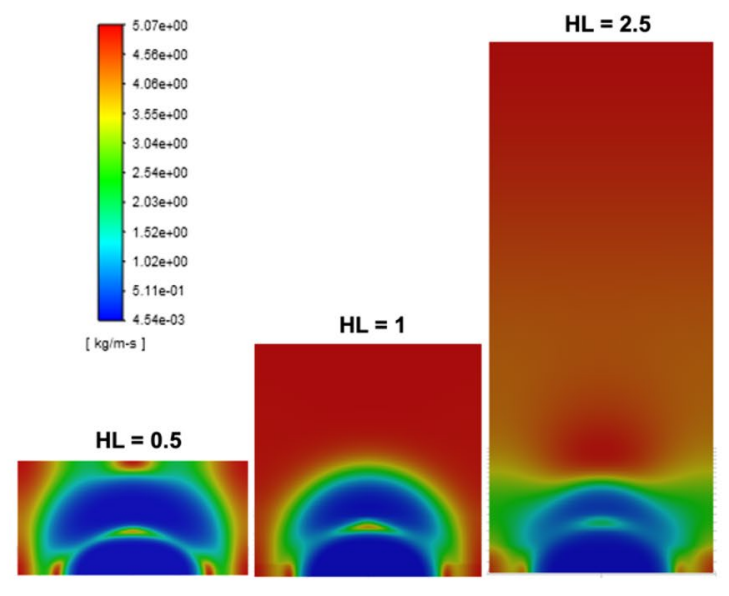

(d)

cosity contour plot textured microchannels with different constriction ratios at $\mathrm{Cu}=3$ across a single post

Recently, the thickness of the depletion layer is shown to be minimised at critical applied shear rates for the shearthinning liquids (Koponen et al. 2019; Turpeinen et al. 2020). At these critical applied shear rates, the contribution of slip to the flow enhancement becomes dominant. Consequently, we estimated the thickness of the depletion layer $(d)$ on the $\mathrm{SH}$-textured surfaces from the numerical simulations as (Koponen et al. 2019):

$d=\frac{u_{s} \eta_{w}}{\tau_{w}\left(1-\frac{\eta_{w}}{\eta_{b}}\right)}$.

Here, $u_{\mathrm{s}}$ is the slip velocity at the wall, $\eta_{\mathrm{w}}$ is the viscosity at the wall, $\eta_{\mathrm{b}}$ is the viscosity of the bulk liquid far 


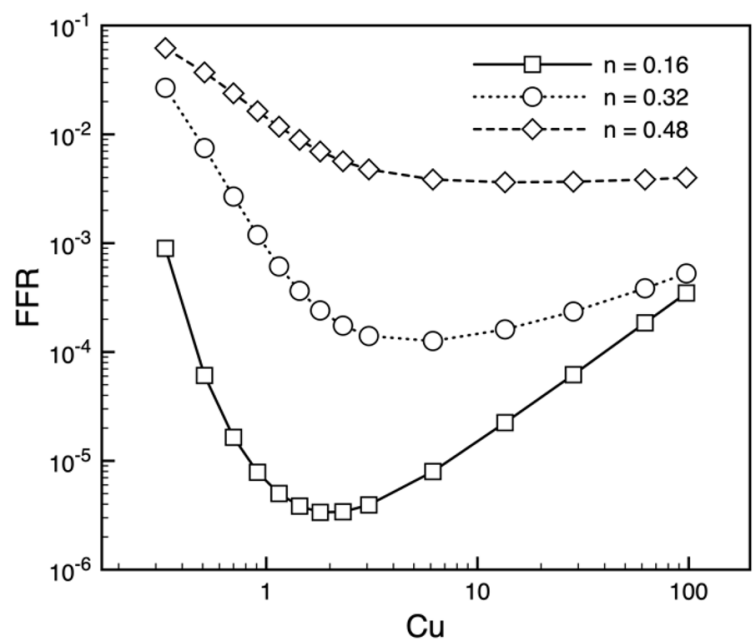

(a)

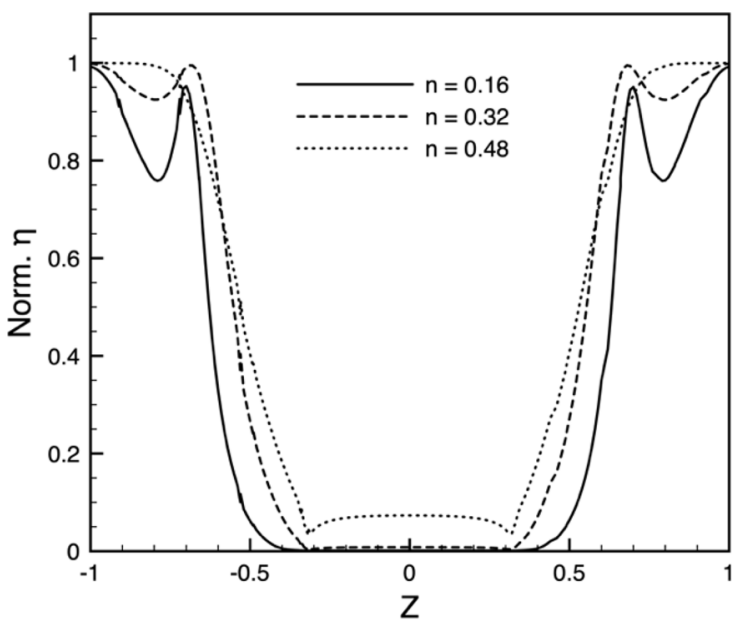

(c)

Fig. 13 Friction factor ratio as a function of Carreau number for shear-thinning liquids with different power-law exponents flowing in textured microchannels containing a longitudinal ribs and b posts. c The viscosity variation normal to the flow direction for a single post

away from the wall, and $\tau_{\mathrm{w}}$ is the wall shear stress. By taking $\tau_{\mathrm{w}}=\eta_{\mathrm{w}} \gamma_{\mathrm{w}}$, where $\gamma_{\mathrm{w}}$ is the shear rate at the wall, the thickness of the depletion layer is evaluated for a flow past microchannels with transverse ribs at different constriction ratios $(\mathrm{HL}=1$ and 2.5), gas fractions $(\mathrm{GF}=0.5$ and 0.9$)$ and power-law exponents $(n=0.16$ and 0.32$)$. Here, the microchannel heights corresponding to $\mathrm{HL}=1$ and 2.5 are 200 and $500 \mu \mathrm{m}$, respectively. The thickness of the depletion layer for a flow past transverse ribs arranged at a $\mathrm{GF}=0.5$ in a microchannel with a height of $100 \mu \mathrm{m}$ is estimated to be $\sim 2.5 \mu \mathrm{m}$.

Figure 16b shows the non-monotonic variation of the $\eta$. ratio with the Carreau number when the depletion layer thickness is $2.5 \mu \mathrm{m}$. Whereas, such behavior is no longer appears when the thickness of the depletion layer

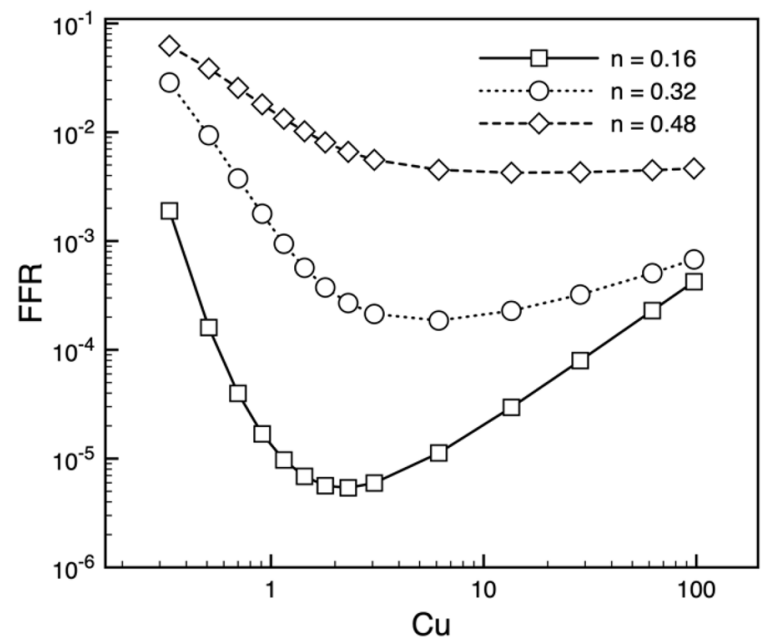

(b)

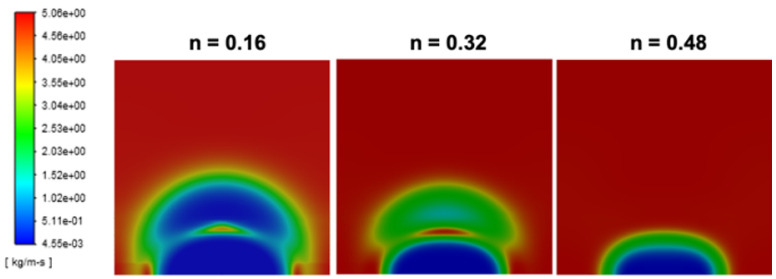

(d)

in textured microchannel $(\mathrm{HL}=1)$ at $y / H=0$ fed with shear-thinning liquids having different power-law exponents at $\mathrm{Cu}=3$. d The viscosity contours across a single post in textured microchannels $(\mathrm{HL}=1$ and $\mathrm{Cu}=3$ )

is increased to $5 \mu \mathrm{m}$. Therefore, it is apparent that locating the depletion layer is essential to capture the viscosity disturbances inside the microchannel. To further understand the behavior of the depletion layer, the variation of its thickness at different Carreau number is estimated as shown in Fig. 16c. As can be seen, the thickness of the depletion layer indeed becomes minimum at $\mathrm{Cu}=2.3$ irrespective of the constriction ratio and shifts towards the higher Carreau number by increasing the power-law exponent. Consequently, when the depletion layer thickness minimises, the hydrodynamic drag reduces. Therefore, it is evident that the depletion layer plays an important role in the slip flow of shear-thinning liquid on SH-textured 


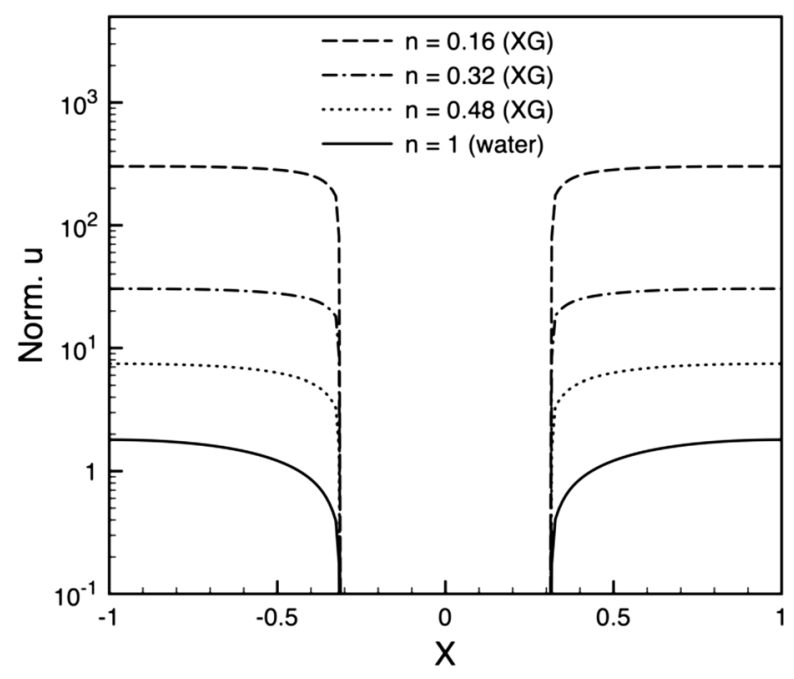

(a)

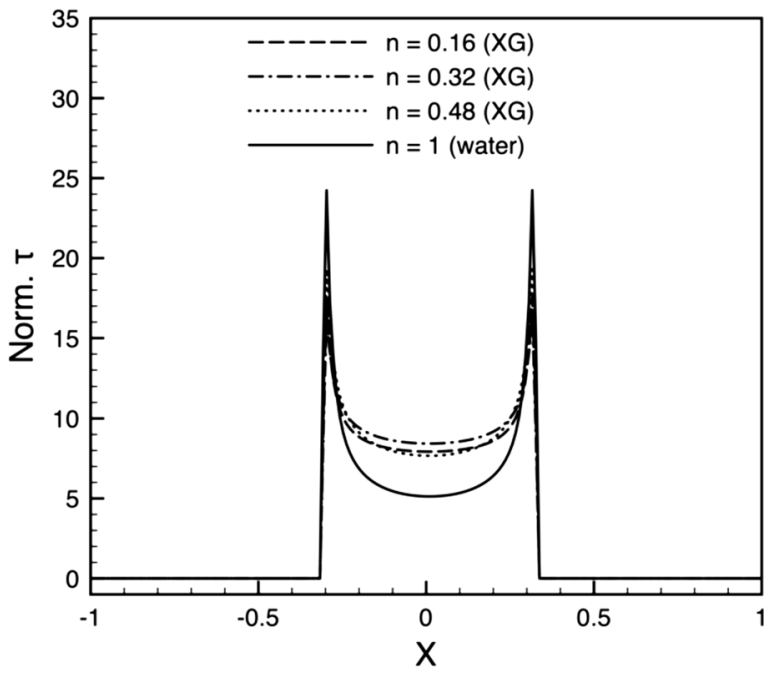

(b)

water. The microchannel constriction ratio here is $\mathrm{HL}=1$ and $\mathrm{GF}=0.9$. The liquids are flowing at $\mathrm{Cu}=1.15$
Fig. 14 a The normalized interfacial velocity profiles and $\mathbf{b}$ the normalized interfacial shear stress profiles for flow past posts obtained for shear-thinning liquids with different power-law exponents and

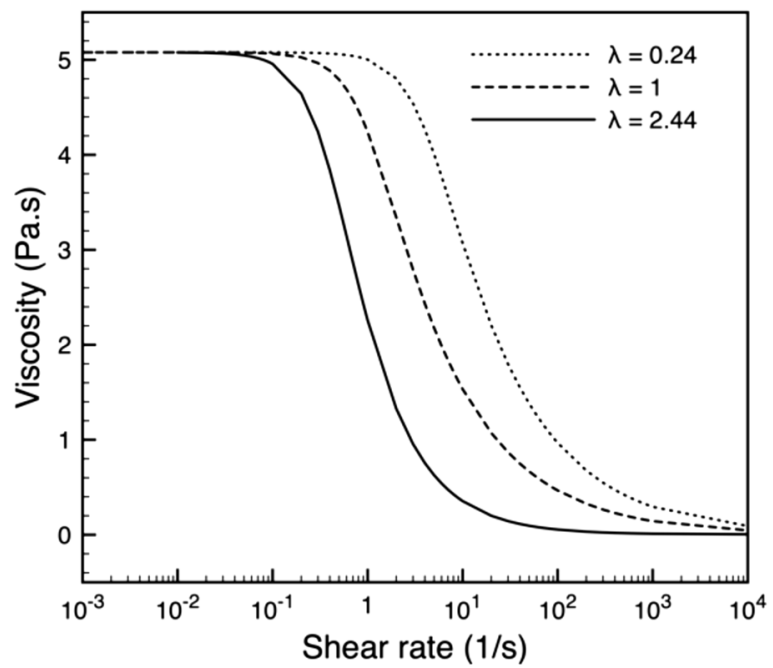

(a)

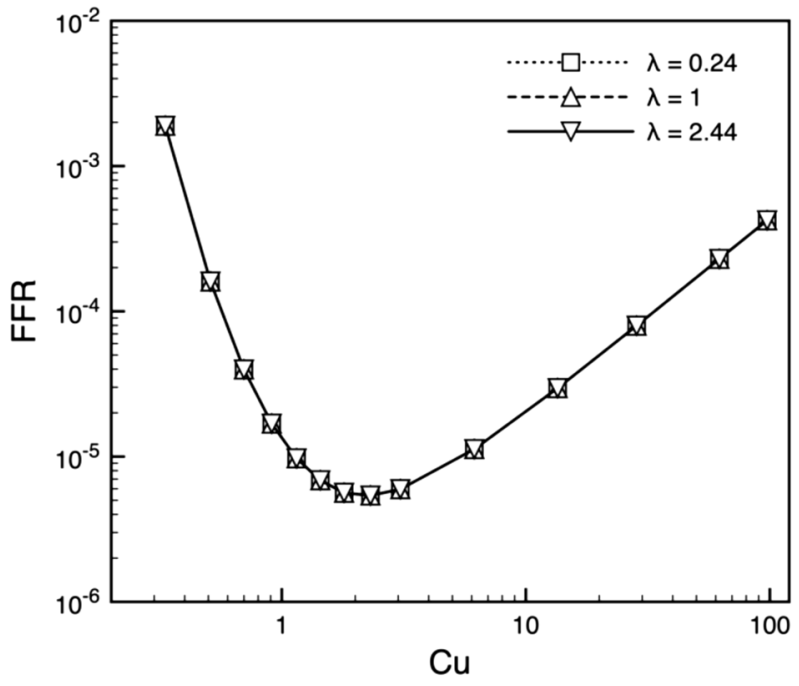

(b)

Fig. 15 a The viscosity as a function of shear rate at different relaxation times. b The friction factor ratio for a flow of shear-thinning liquids with different relaxation times in microchannels with posts. Here $\mathrm{HL}=1$ and GF $=0.9$

surfaces. It should be also noted that the determination of depletion layer thickness depends on the velocity profile fitting inside the channels (Turpeinen et al. 2020). However, such exercise is outside the scope of this research and therefore, further work is warranted in this direction especially in the case of 3D flows inside the microchannels.

\section{Flow enhancement in super-hydrophobic textured microchannels}

To further validate the numerical results, water and shearthinning liquids (XG1, XG2 and XG5) were fed through the smooth and SH-textured microchannels with different 


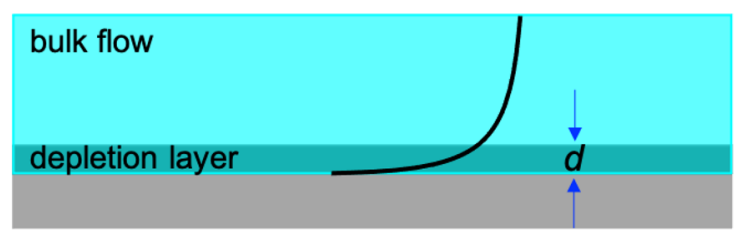

(a)

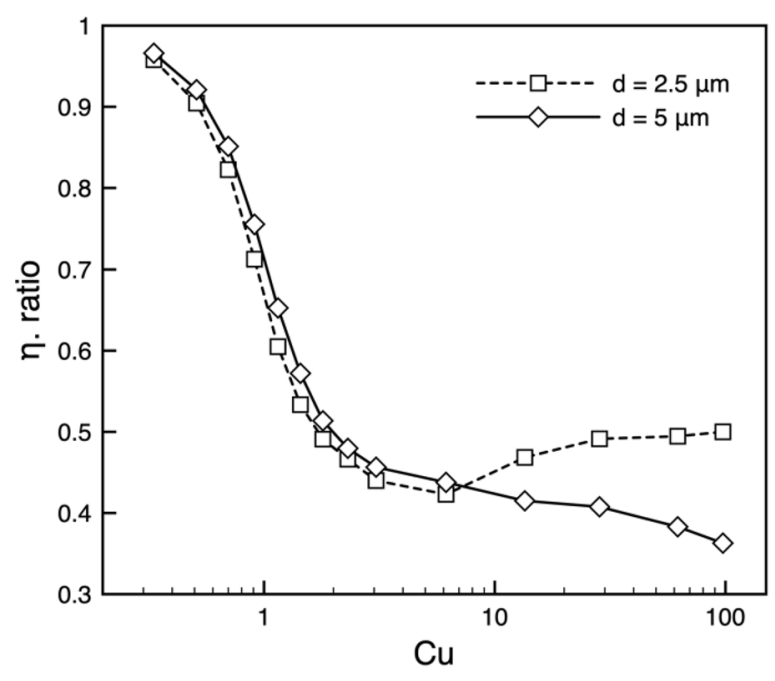

(b)

Fig. 16 a An illustration of depletion layer and bulk layer on the surface. $\mathbf{b}$ The viscosity ratio of the depletion and bulk layers as a function of Carreau number and depletion layer thickness. Here $\mathrm{HL}=1$,

heights and the flow enhancement $(\varepsilon)$ was estimated using Eq. 4:

$\varepsilon=\frac{\varepsilon_{t}}{\varepsilon_{s}}-1$.

Here, $\varepsilon_{s}$ and $\varepsilon_{t}$ are the flow rates in the smooth and $\mathrm{SH}$-textured microchannels, respectively. The microchannel heights investigated in the experiments are $90 \mu \mathrm{m}$ and $200 \mu \mathrm{m}$ correspond to the microchannel constriction ratios of $\mathrm{HL}=0.45$ and $\mathrm{HL}=1$, respectively. Furthermore, the $\mathrm{SH}$-textured surfaces were fabricated in such a way that the gas fraction for both ribs and posts are $85 \%$ to have a fair comparison. To validate the microchannel configuration adopted in this research, first the smooth microchannels were fabricated both the sides. Then, the equal volume flow rates from the two outlets were ensured for each flow rate at the inlet.

Figure 17 shows exemplary images of the quantity of liquid obtained in the smooth (right) and SH-textured (left) microchannels with a height of $90 \mu \mathrm{m}$ for the XG liquids. Here, the microchannels were fed with a flow rate of 8.33 $\mathrm{mm}^{3} / \mathrm{s}$. The flow rate obtained with the XG2 liquid in the

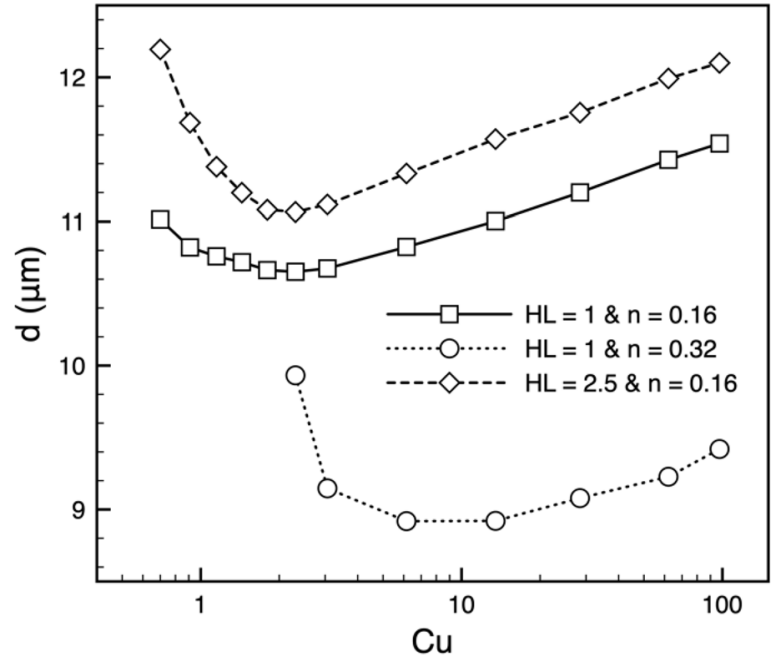

(c)

$\mathrm{GF}=0.5$ and $n=0.16$. $\mathrm{c}$ The depletion layer thickness as a function of Carreau number. Only the microchannels with transverse ribs are considered here

smooth and SH-textured microchannels $2.5 \mathrm{~mm}^{3} / \mathrm{s}$ and $5.55 \mathrm{~mm}^{3} / \mathrm{s}$, respectively. Whereas, smooth and SH-textured microchannels resulted in a flow rate of $1.94 \mathrm{~mm}^{3} / \mathrm{s}$ and $6.11 \mathrm{~mm}^{3} / \mathrm{s}$, respectively, for the XG5 liquid.

Figure 18a, b quantifies the flow enhancement for the smooth and SH-textured microchannels when fed with water and XG liquids for posts and transverse ribs. As shown, the flow enhancement due to the SH textures is constant for the flow of water with respect to the smooth microchannels. While the posts resulted in a flow enhancement of 0.4-0.56, whereas the transverse ribs showed an enhancement of $0.27-0.32$ when the water is fed through the microchannels. On the other hand, the flow enhancement increases up to a flow rate of $0.83 \mathrm{~mm}^{3} / \mathrm{s}$ and decreases thereafter for the XG2 and XG5 liquids in the microchannel with $\mathrm{HL}=0.45$ for both the posts and transverse ribs. Since the XG5 liquid is strongly shear-thinning $(n=0.16)$ than the XG2 liquid $(n=0.31)$, the flow enhancement is found to be more in the former than the latter. For both liquids, the flow enhancement curves are plateauing towards the limit set by the water at low and high flow rates. It should also be noted that the maximum flow enhancement is at least 20 times that of the water when the XG5 liquid is employed in the case of 
Fig. 17 Images showing flow rate enhancement in the $\mathrm{SH}$ textured microchannels with posts (left) compares to the smooth microchannels (right) for a XG2, b XG5 liquids. Here the microchannel height is $90 \mu \mathrm{m}$ and the flow rate is 8.33 $\mathrm{mm}^{3} / \mathrm{s}$

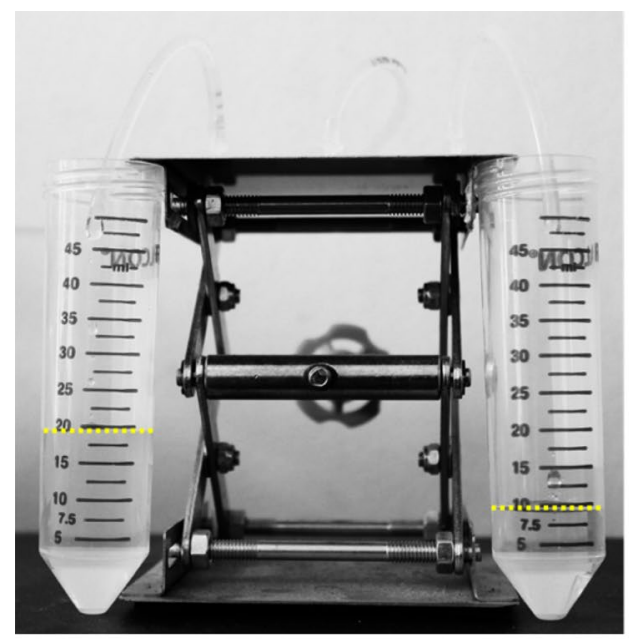

(a)

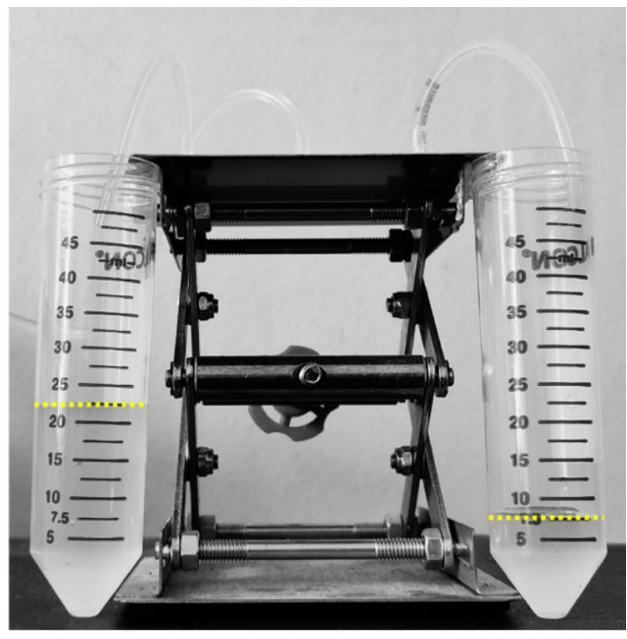

(b)

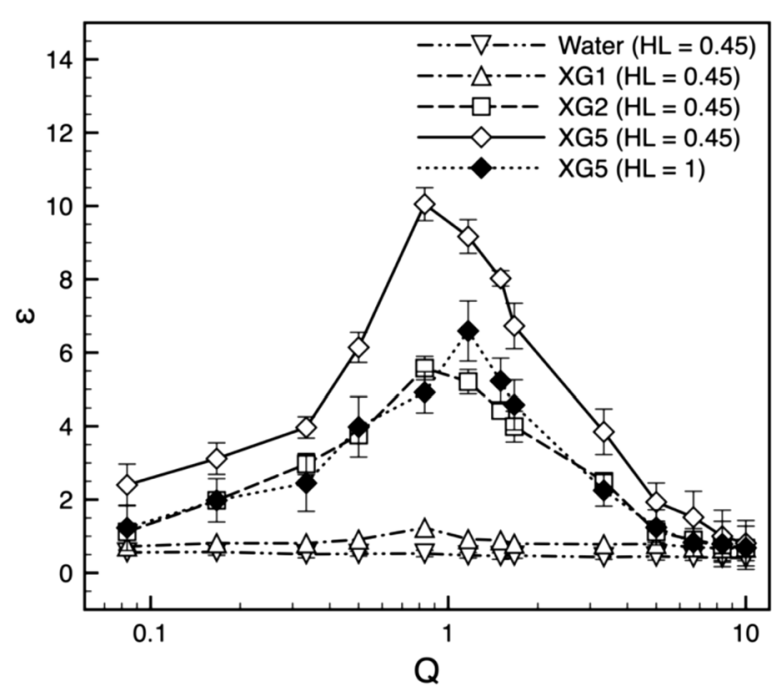

(a)

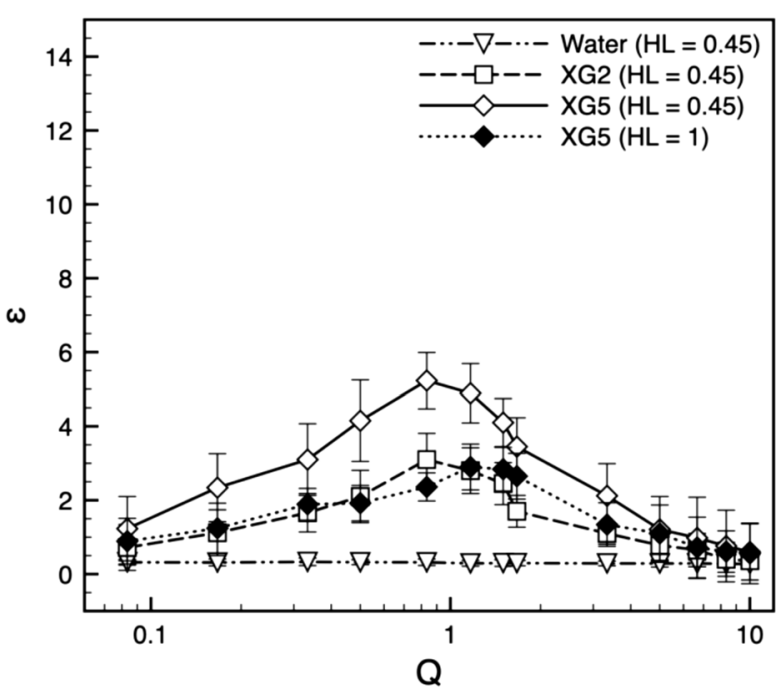

(b)

Fig. 18 The flow enhancement in microchannels with $2 \mathrm{H}=90 \mu \mathrm{m}(\mathrm{HL}=0.45)$ and $200 \mu \mathrm{m}(\mathrm{HL}=1)$ and decorated with a posts and $\mathbf{b}$ transverse ribs as a function of the flow rate $\left(\mathrm{mm}^{3} / \mathrm{s}\right)$ for water and $\mathrm{XG}$ liquids

posts. At the same time, the transverse ribs exhibited less enhancement than the posts, which is in corroboration with the numerical results. However, the flow enhancement is noticed to be insignificant in the case of XG1 liquid for both posts and transverse ribs, although it was marginally higher than the water in the former. This could be attributed to the weakly shear-thinning $(n=0.47)$ nature of the XG1 liquid. When the height of the microchannels is increased corresponding to $\mathrm{HL}=1$, the magnitude of the flow enhancement is decreased by $40 \%$ for the XG5 liquid, which also qualitatively corroborates the numerical estimates (see Fig. 10). At the same time, the maxima of the flow enhancement is also shifted to a higher inlet flow rate. This is because a relatively high shear rate is needed to obtain the maximum flow enhancement (or a minimum friction factor ratio) in microchannels with a high constriction ratio than those with a low constriction ratio.

\section{Conclusion}

Super-hydrophobic textured surfaces are known to exhibit hydrodynamic drag reduction properties when Newtonian liquids are employed both in external and internal flow settings. In this work, the flow behaviour of the shearthinning liquids in the pressure-driven flow through the 
super-hydrophobic textured microchannels in investigated in detail. The friction factor ratio, which signifies the hydrodynamic drag reduction, is estimated as a function of geometric, flow and fluid parameters through numerical simulations. The friction factor ratio exhibited non-monotonic variation with the Carreau number on one- and bi-dimensional surface textured irrespective of the microchannel constriction ratios. The stronger the shear-thinning liquid lesser the friction factor ratio. The super-hydrophobic dual-scale topographies fabricated by the femtosecond laser are used to prepare the textured microchannels. Aqueous Xanthan Gum liquids when fed through such superhydrophobic textured microchannels containing posts and ribs led to a huge increase in the flow enhancement when compared to water. The experimental results were qualitatively in agreement with the numerical findings.

In this research, we considered liquid-gas interface to be a shear-free boundary. However, the circulation in the gas cavities could affect the local slip velocity for the shear-thinning liquids similar to the Newtonian liquids and thus the friction factor. Consequently, the geometry and size of the underlying gas cavities is another important factor to consider in the numerical modelling. At the same time, given the importance of lubricant-impregnated textured surfaces in the context of robust functional surfaces in a range of applications when compared to the gas-cushioned textured surfaces, our future studies will focus on understanding the interaction of shear-thinning liquids with such surfaces. The elucidated flow behaviour of shear-thinning liquids in superhydrophobic textured microchannels in this work not only relevant to microfluidic devices but also indirectly significant to the food processing and pharmaceutical industries. Especially, when employing the automated cleaning-in-place processes are adopted to clear the viscoelastic deposits on vessel surfaces and pumping viscoelastic liquids through the pipelines. In such internal and external flow settings, the usage of superhydrophobic textures on walls could minimise the pumping power requirements and reduce the water usage requirements, respectively. In addition, the ultrafast lasers to fabricate drag reducing superhydrophobic surfaces is also demonstrated in this work. The dual-scale structures are fabricated at a rate of $2 \mathrm{~mm}^{2} / \mathrm{s}$ on metallic sheets, but much higher processing speed can be achieved with high dynamics scan head and laser sources. Therefore, ultrafast laser processing can be adopted to fabricate superhydrophobic periodic textures rapidly over large areas and on $3 \mathrm{D} /$ freeform surfaces where the drag reduction properties are needed, right from microfluidics to marine vehicles.

Supplementary Information The online version contains supplementary material available at https://doi.org/10.1007/s10404-021-02470-7.
Acknowledgements The research work is conducted within the framework of the UKIERI-DST programme on "Surface functionalisation for food, packaging, and healthcare applications" and the European Commission H2020 project "High-impact injection moulding platform for mass-production of 3D and/or large micro- structured surfaces with antimicrobial, self-cleaning, anti-scratch, anti-squeak and aesthetic functionalities" (HIMALAIA).

Open Access This article is licensed under a Creative Commons Attribution 4.0 International License, which permits use, sharing, adaptation, distribution and reproduction in any medium or format, as long as you give appropriate credit to the original author(s) and the source, provide a link to the Creative Commons licence, and indicate if changes were made. The images or other third party material in this article are included in the article's Creative Commons licence, unless indicated otherwise in a credit line to the material. If material is not included in the article's Creative Commons licence and your intended use is not permitted by statutory regulation or exceeds the permitted use, you will need to obtain permission directly from the copyright holder. To view a copy of this licence, visit http://creativecommons.org/licenses/by/4.0/.

\section{References}

Brunchi CE, Bercea M, Morariu S, Dascalu M (2016) Some properties of xanthan gum in aqueous solutions: effect of temperature and pH. J Polym Res 23(7):1-8

Chen C, Weng D, Mahmood A, Chen S, Wang J (2019) Separation mechanism and construction of surfaces with special wettability for oil/water separation. ACS Appl Mater Interfaces 11(11):11006-11027

Chen J, Kim YJ, Hwang WR (2020) General criteria for the estimation of effective slip length over corrugated surfaces. Microfluid Nanofluid 24(9):1-15

Cheng YP, Teo CJ, Khoo BC (2009) Microchannel flows with superhydrophobic surfaces: effects of Reynolds number and pattern width to channel height ratio. Phys Fluids 21(12):122004

Cowley A, Maynes D, Crockett J (2016) Inertial effects on thermal transport in superhydrophobic microchannels. Int J Heat Mass Transf 101:121-132

Crowdy D (2017) Effect of shear thinning on superhydrophobic slip: perturbative corrections to the effective slip length. Phys Rev Fluids 2(12): 124201

Davis AM, Lauga E (2009) Geometric transition in friction for flow over a bubble mattress. Phys Fluids 21(1):1701

De Lemos MJ (2012) Turbulence in porous media: modeling and applications. Elsevier, Amsterdam

Dilip D, Kumar SV, Bobji MS, Govardhan RN (2018) Sustained drag reduction and thermo-hydraulic performance enhancement in textured hydrophobic microchannels. Int J Heat Mass Transf 119:551-563

Elberskirch L, Knoll T, Moosmann A, Wilhelm N, von Briesen H, Wagner S (2019) A novel microfluidic mucus-chip for studying the permeation of compounds over the mucus barrier. J Drug Deliv Sci Technol 54:101248

Gaddam A, Garg M, Agrawal A, Joshi SS (2015) Modeling of liquidgas meniscus for textured surfaces: effects of curvature and local slip length. J Micromech Microeng 25(12):125002

Gaddam A, Agrawal A, Joshi SS, Thompson MC (2018) Slippage on a particle-laden liquid-gas interface in textured microchannels. Phys Fluids 30(3):032101 
Gaddam A, Sharma H, Karkantonis T, Dimov S (2021) Anti-icing properties of femtosecond laser-induced nano and multiscale topographies. Appl Surf Sci 552:149443

Game SE, Hodes M, Keaveny EE, Papageorgiou DT (2017) Physical mechanisms relevant to flow resistance in textured microchannels. Phys Rev Fluids 2(9):094102

Geyer F, D’Acunzi M, Sharifi-Aghili A, Saal A, Gao N, Kaltbeitzel A, Sloot TF, Berger R, Butt HJ, Vollmer D (2020) When and how self-cleaning of superhydrophobic surfaces works. Sci Adv 6(3):eaaw9727

Haase AS, Wood JA, Lammertink RG, Snoeijer JH (2016) Why bumpy is better: the role of the dissipation distribution in slip flow over a bubble mattress. Phys Rev Fluids 1(5):101

Haase AS, Wood JA, Sprakel LM, Lammertink RG (2017) Inelastic non-Newtonian flow over heterogeneously slippery surfaces. Phys Rev E 95(2):023105

Huang L, Yao Y, Peng Z, Zhang B, Chen S (2021) How to Achieve a monostable cassie state on a micropillar-arrayed superhydrophobic surface. J Phys Chem B 125(3):883-894

Hwang GB, Page K, Patir A, Nair SP, Allan E, Parkin IP (2018) The anti-biofouling properties of superhydrophobic surfaces are shortlived. ACS Nano 12(6):6050-6058

Javaherchian J, Moosavi A (2019) Pressure drop reduction of powerlaw fluids in hydrophobic microgrooved channels. Phys Fluids 31(7):073106

Jiang R, Hao L, Song L, Tian L, Fan Y, Zhao J, Liu C, Ming W, Ren L (2020) Lotus-leaf-inspired hierarchical structured surface with non-fouling and mechanical bactericidal performances. Chem Eng J 398:609

Kant K, Pitchumani R (2021) Laminar drag reduction in microchannels with liquid infused textured surfaces. Chem Eng Sci 230:116196

Ko YS, Kim HJ, Ha CW, Lee C (2020) Quantifying frictional drag reduction properties of superhydrophobic metal oxide nanostructures. Langmuir 36(40):11809-11816

Kojić S, Birgermajer S, Radonić V, Podunavac I, Jevremov J, Petrović B, Marković E, Stojanović GM (2020) Optimization of hybrid microfluidic chip fabrication methods for biomedical application. Microfluid Nanofluid 24(9):1-12

Koponen A, Haavisto S, Salmela J, Kataja M (2019) Slip flow and wall depletion layer of microfibrillated cellulose suspensions in a pipe flow. Annu Trans Nord Soc Rheol 27:13-20

Lauga E, Stone HA (2003) Effective slip in pressure-driven Stokes flow. J Fluid Mech 489:55

Laxmi V, Tripathi S, Joshi SS, Agrawal A (2020) Separation and enrichment of platelets from whole blood using a pdms-based passive microdevice. Ind Eng Chem Res 59(10):4792-4801

Li Y, Alame K, Mahesh K (2017) Feature-resolved computational and analytical study of laminar drag reduction by superhydrophobic surfaces. Phys Rev Fluids 2(5):054002

Li H, Li Z, Tan X, Wang X, Huang S, Xiang Y, Lv P, Duan H (2020) Three-dimensional backflow at liquid-gas interface induced by surfactant. J Fluid Mech 899

Liu W, Ren Y, Tao Y, Zhou Z, Wu Q, Xue R, Yao B (2020) Multiple frequency electrothermal induced flow: theory and microfluidic applications. J Phys D: Appl Phys 53(17):175304

Liu W, Tao Y, Ge Z, Zhou J, Xu R, Ren Y (2021) Pumping of electrolyte with mobile liquid metal droplets driven by continuous electrowetting: a full-scaled simulation study considering surface-coupled electrocapillary two-phase flow. Electrophoresis 42(7-8):950-966

Lobaton EJ, Salamon TR (2007) Computation of constant mean curvature surfaces: application to the gas-liquid interface of a pressurized fluid on a superhydrophobic surface. J Colloid Interface Sci 314(1):184-198
Malkin AY, Patlazhan SA (2018) Wall slip for complex liquids-phenomenon and its causes. Adv Coll Interface Sci 257:42-57

Maynes D, Jeffs K, Woolford B, Webb BW (2007) Laminar flow in a microchannel with hydrophobic surface patterned microribs oriented parallel to the flow direction. Phys Fluids 19(9):093603

Patlazhan S, Vagner S (2017) Apparent slip of shear thinning fluid in a microchannel with a superhydrophobic wall. Phys Rev E 96(1):013104

Peaudecerf FJ, Landel JR, Goldstein RE, Luzzatto-Fegiz P (2017) Traces of surfactants can severely limit the drag reduction of superhydrophobic surfaces. Proc Natl Acad Sci 114(28):7254-7259

Philip JR (1972) Flows satisfying mixed no-slip and no-shear conditions. Z Für Angew Math Phys ZAMP 23(3):353-372

Raoufi MA, Mashhadian A, Niazmand H, Asadnia M, Razmjou A, Warkiani ME (2019) Experimental and numerical study of elasto-inertial focusing in straight channels. Biomicrofluidics 13(3):034103

Samaha MA, Vahedi Tafreshi H, Gad-el-Hak M (2011) Modeling drag reduction and meniscus stability of superhydrophobic surfaces comprised of random roughness. Phys Fluids 23(1):012001

Sharma H, John K, Gaddam A, Navalkar A, Maji SK, Agrawal A (2018) A magnet-actuated biomimetic device for isolating biological entities in microwells. Sci Rep 8(1):1-14

Sharma H, Gaddam A, Agrawal A, Joshi SS (2019) Slip flow through microchannels with lubricant-infused bi-dimensional textured surfaces. Microfluid Nanofluid 23(2):28

Sharma H, Gaddam A, Agrawal A, Joshi SS, Dimov SS (2020) Influence of texture shape and arrangement on thermo-hydraulic performance of the textured microchannels. Int J Therm Sci 147:6146

Shende T, Niasar VJ, Babaei M (2021) Effective viscosity and Reynolds number of non-Newtonian fluids using Meter model. Rheol Acta 60(1):11-21

Siddiquie RY, Gaddam A, Agrawal A, Dimov SS, Joshi SS (2020) Anti-biofouling properties of femtosecond laser-induced submicron topographies on elastomeric surfaces. Langmuir 36(19):5349-5358

Teo CJ, Khoo BC (2009) Analysis of Stokes flow in microchannels with superhydrophobic surfaces containing a periodic array of micro-grooves. Microfluid Nanofluid 7(3):353

Tsai P, Peters AM, Pirat C, Wessling M, Lammertink RG, Lohse D (2009) Quantifying effective slip length over micropatterned hydrophobic surfaces. Phys Fluids 21(11):112002

Turpeinen T, Jäsberg A, Haavisto S, Liukkonen J, Salmela J, Koponen AI (2020) Pipe rheology of microfibrillated cellulose suspensions. Cellulose 27(1):141-156

Winter HH (1987) Viscous dissipation term in energy equations. Calc Measur Tech Momentum Energy Mass Transfer 7:27-34

Wu H, Yang Z, Cao B, Zhang Z, Zhu K, Wu B, Jiang S, Chai G (2017) Wetting and dewetting transitions on submerged superhydrophobic surfaces with hierarchical structures. Langmuir 33(1):407-416

Ybert C, Barentin C, Cottin-Bizonne C, Joseph P, Bocquet L (2007) Achieving large slip with superhydrophobic surfaces: Scaling laws for generic geometries. Phys Fluids 19(12):123601

Zhong L, Oostrom M, Truex MJ, Vermeul VR, Szecsody JE (2013) Rheological behavior of xanthan gum solution related to shear thinning fluid delivery for subsurface remediation. J Hazard Mater 244:160-170

Publisher's Note Springer Nature remains neutral with regard to jurisdictional claims in published maps and institutional affiliations. 